\title{
A Review on Accelerometry Based Gait Analysis and Emerging Clinical Applications
}

\author{
Delaram Jarchi, James Pope, Tracey K. M. Lee, Larisa Tamjidi, Amirhosein Mirzaei and Saeid Sanei
}

\begin{abstract}
Gait analysis continues to be an important technique for many clinical applications to diagnose and monitor certain diseases. Many mental and physical abnormalities cause measurable differences in a person's gait. Gait analysis has applications in sport, computer games, physical rehabilitation, clinical assessment, surveillance, human recognition, modelling, and many other fields. There are established methods using various sensors for gait analysis, of which, accelerometers are one of the most often employed. Accelerometer sensors are generally more user friendly and less invasive. In this paper, we review research regarding accelerometer sensors used for gait analysis with particular focus on clinical applications. We provide a brief introduction to accelerometer theory followed by other popular sensing technologies. The commonly used gait phases and parameters are enumerated. The details of extracting the papers for review are provided. We also review several gait analysis software. Then, we provide an extensive report of accelerometry based gait analysis systems and applications with additional emphasis on trunk accelerometry. We conclude this review with future research directions.
\end{abstract}

Index Terms-accelerometer, gait, inertial measurement unit, trunk

\section{INTRODUCTION}

Despite the appearance of people, their shape, or their hair and skin colours, there are many other factors used in describing them and their actions. Among these factors those often called biometrics include gait, facial features, finger print, voice, iris, and other particular markers such as skin spots. Gait includes static features such as height, stride length, and silhouette bounding box lengths plus some dynamic features such as the time-frequency domain parameters [1]. It can be characterised through the information from various systems including ambient and wearable systems such as video and accelerometer [2].

A useful demarcation between the types of gait analyses has been presented in [1]. The study of human gait was traditionally performed in medical diagnosis. It is a subject within biomechanic, kinesiology, or other medical fields like podiatry. This field of study has provided much of the terminology used in gait analysis as well as the initial experiments on recognition.

D. Jarchi is with the Department of Engineering Science, University of Oxford, Oxford, OX3 7DQ, UK (delaram.jarchi@eng.ox.ac.uk). J. Pope is with the Electrical and Electronic Engineering department, University of Bristol, Bristol, BS8 1UB, UK (james.pope @ bristol.ac.uk). T. K. M. Lee is with the School of Electrical and Electronic Engineering, Singapore Polytechnic, 500 Dover Rd. L (tlee@sp.edu.sg). L. Tamjidi is with the Department of Mathematics, University of New Brunswick, Fredericton, New Brunswick, Fredericton, E3B 5A3, Canada (L.tamjidi@unb.ca). A. Mirzaei is with Islamic Azad Univesrity of Shabestar (a.mirzaei@iaushab.ac.ir) and S. Sanei is with School of Science and Technology, Nottingham Trent University, Nottingham, NG11 8NF, UK (saeid.sanei@ntu.ac.uk).
Biomechanical and clinical studies demonstrate that the combined actions of hundreds of joints and muscles can be used to represent each person with a unique way of walking. In addition, gait can reveal the presence of certain sicknesses, moods and can distinguish gender differences [3]. It has been shown that variability of gait for a healthy person is fairly consistent and not easily changed, while allowing for differentiation with others.

On the other hand, physical and mental generative or degenerative disabilities and diseases are well characterised by significant changes in body mostly hands and feet movements. Many of these abnormalities stem from brain diseases directly affecting the neurons in the motor area of the brain. This consequently influences the gait dynamics and morphology. Amyotrophic lateral sclerosis (ALS) and Ataxia both refer to neurological diseases [4] that influence the movement and control of posture and balance resulting in poor coordination. Some brain disorders, such as attention deficit hyperactivity disorder (ADHD), autism, dementia (such as Alzheimer), Parkinson's disease (PD), depression, Pick's disease, or bipolar disorder, do not directly target motor area but instead slow down the body motion and indirectly affect hand and foot movements. This can happen to many diseases affecting the central nervous system (CNS). On the other hand, a tumour in the brain, no matter benign or cancerous, very likely affects the brain motor area and can laterally or fully paralyze the subject [4].

Among many others, cerebral palsy also affects muscle control and movement. Cerebrovascular diseases can block the food and oxygen supply to parts of the brain and lead to stroke. All these diseases directly affect the gait.

Creutzfeldt-Jakob Disease (CJD), often called mad cow disease [5], and Huntington's disease [6], cause gradual death of the brain cells and can change the subject's mood and consequently, their normal movements. They start with occasional mood or mental disabilities [6] and follow by a lack of coordination and an unsteady movement [7].

Encephalomyelitis, referring to inflammation of the brain or spinal cord, can affect the gait and body movement severely. Another similar disease is meningitis where the inflammation can cause damage to the brain and spinal cord.

Multiple sclerosis (MS) is a degenerative disease damaging the myelin which is the covering layer of the nerve cells in the brain or spinal cord [8]. This damage disrupts the communications between the cells in the nervous system resulting in a range of signs and symptoms [9], including lack of coordination [8].

In many cases of paralysis, an injury in the middle or lower regions of the spinal cord is likely to disrupt any 
function below the injury point, resulting in the inability to move the feet or feel sensations. In this situation, the brain is unable to relay a signal to an area of the body. Also, in the case of brain diseases such as epilepsy, seizure onset will result in temporary loss of control often, but not always, accompanied by convulsions, unconsciousness or uncontrolled jerking movement (tonic-clonic seizure) to as subtle as a momentary loss of awareness, namely absence seizures [10]. Analysis of gait for fall detection can significantly reduce the risk of injury for such patients.

In using video cameras for gait often more than one camera is needed to overcome the occlusion problem, therefore, it is more suitable for indoor use. Presently, rehabilitative assessment and monitoring are labour intensive.

One way to emulate and translate the experience of healthcare practitioners is in the form of tests for limb function for tasks deemed essential in the activities of daily living (ADL). There are a large number of such established tests which involve the movements of a patient and their interactions with their environment [11]. The use of sensors in consumer devices such as mobile phones and gaming consoles allows for a better user experience as the processors in these devices deduce the intention of the user by their movements.

Of the sensors that are currently used to measure and detect movement, the accelerometer is commonly adopted. Its widespread use initially came about from automotive applications such as activation of airbags. Presently by itself or as part of an Inertial Measurement Unit (IMU), accelerometers find applications in consumer devices such as digital cameras, health trackers, smartphones and interactive computer games. In less prominent applications, accelerometers are used in demanding industrial environments, such as measuring vibrations between motors and their mountings and for measuring tilt. All these require accelerometers to provide higher sensitivity, robustness, and smaller size at a lower cost. As a result, accelerometers have proven very useful for in vivo movement analysis.

There are other considerations related to the sensor deployment, for example, the placement and method of attachment for optimal analysis and also finding the best ways to filter and analyse the data. Commonly used accelerometer types are piezoelectric, thermal and capacitive, the last one is usually fabricated using micro electromechanical sensor (MEMS) technology which is also the lowest in cost because of so called economies of scale. Currently, most work in simulation of accelerometers has to do with verifying a design at the semiconductor fabrication level. There are also some simulations performed at the device level, but many of these treat the device as a "black box".

There are three major areas of challenges that accelerometers present in their use. First one is the effect of drift or change in the internal mechanical or electrical properties, which can manifest itself as a bias or an offset in readings. The second is noise from amplified microscopic mechanical motions. Third is the effect of gravity, which is ever present. While this is strictly not a defect, we have to consider that the gravitational force vector is projected and superimposed along the axes of sensitivity of the accelerometer. Thus, the movements along these axes experience the confounding gravitational effect. Furthermore, with movements at frequencies beyond 10 $\mathrm{kHz}$, the internal mechanical parts of an accelerometer move nonlinearly resulting in dynamic errors.

There are a number of review papers in the literature which address the use of accelerometers and gyroscopes for gait analysis. These include studies focused on the analysis of running gait [12] and the assessment of standing balance and walking stability in PD patients [13]. The effects of device location, age, and testing protocol using accelerometers in gait measurement have been investigated in [14]. A limited review on accelerometer based gait analysis has been published in [15]. A review on inertial sensors for walking speed estimation has been provided in [16]. Other review papers with a broader purpose using wearable or both wearable/non-wearable sensors are presented in [17] and [18]. The most related review paper to our paper is [19]. The distinction between this paper and our work is that our paper first provides a brief methodology and mathematical background on accelerometer and gyroscopes, followed by a brief explanation of other sensing technologies and a comprehensive overview of gait parameters and events. Then, it is focused on the use of accelerometry based gait analysis regarding validation platforms, clinical applications and trunk accelerometry based systems. The remainder of the paper is structured as follows. In section II, the theory of accelerometer and practical considerations are explained. Then, other popular sensing technologies for gait analysis are briefly explained in Section III. The commonly used gait phases and parameters are briefly described in Section IV. This is followed by describing the method to extract accelerometry based papers for reviewing and introducing several gait analysis software used for accelerometry. Then, different platforms for validation of the accelerometry based gait analysis systems, gait analysis applications, and trunk accelerometry based analysis are reported in Section V. Finally Section VI concludes the paper and summarises the research directions in accelerometry based gait analysis and potential improvements for future studies.

\section{THEORY AND USE OF ACCELEROMETER}

In this section, we present a brief and intuitive description of accelerometer operation followed by a discussion on some practical issues in normal use.

\section{A. Theory and use of accelerometer operation}

The devices mentioned in this review measure acceleration by changes in the internal capacitance of the device. As mentioned in Section I, these devices are typically fabricated using MEMS technology on microstructures built into polysilicon. Some microstructures are fixed and some are movable, suspended from fixed points. By impressing a voltage between them, a capacitive effect arises from the electronic charge stored in these structures which is proportional to the area and the physical distances between these structures. External physical movements cause the distances between the microstructures to change, which in turn result in changes to the capacitance which eventually cause changes to the voltage. 
TOP VIEW

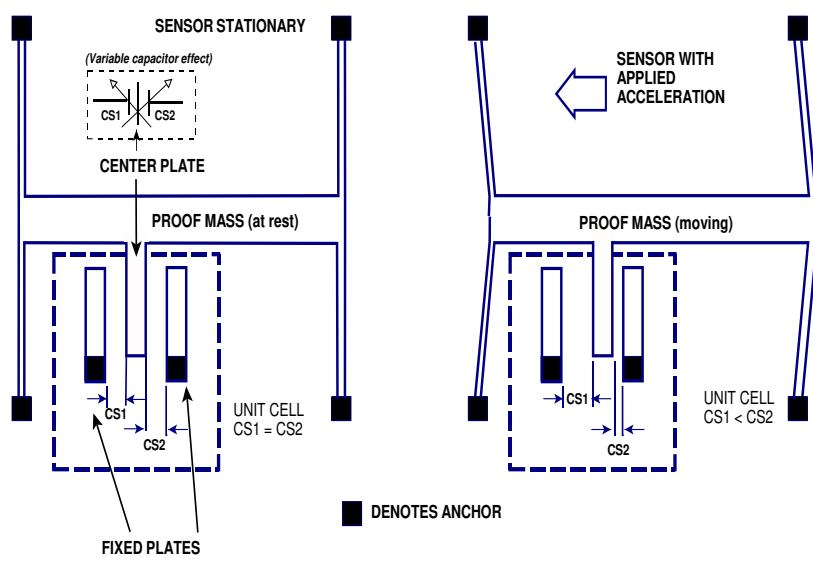

Fig. 1. An accelerometer sensor, simplified view, from ADXL50 [22] datasheet. Anchor points move with frame of the device, while inertia of the proof mass causes the distance between fixed plates to vary, changing the capacitance.

As discussed in Section I, the acceleration has two main components: the first being the inertial acceleration that is linked to changes in velocity, which may result from rotation, translation, or both. The second component consists of gravitational acceleration arising from the microstructures being deflected in proportion to their static orientation to the gravitational field. Removing the confounding effect of this kind of acceleration [20] can be difficult.

In the simplified diagram of Fig. 1 the main interaction happens between a proof mass to which some plates are attached. The proof mass is suspended from the body or frame of the accelerometer device and secured through anchor points. Another set of plates which are fixed to the frame and the changes in the distance between the two induces a change in the voltage between the plates. When the accelerometer frame moves, the inertia of the proof mass induces a reactionary force which is applied to the springs (fabricated from polysilicon). These springs are affixed to the frame of the device at anchor points. The spring deformation is assumed to be linear so that the accelerometer obeys the familiar mass-spring-damper equation derived from Newton's and Hooke's Laws [21]:

$$
M \frac{d^{2} \mathbf{E}}{d t^{2}}=M \frac{d^{2} \mathbf{D}}{d t^{2}}+b \frac{d \mathbf{D}}{d t}+k \mathbf{D}
$$

where $M$ is the mass of the proof mass, $\mathbf{E}, \mathbf{D}$ the distance travelled by the proof mass with respect to the earth and with respect to its anchors respectively, $b$ is the damping factor, and $k$ the spring constant. In addition, there are electrostatic forces between the fixed and moving plates which also need to be considered. In order to measure the speed of movement, consider the relative motion between the stationary plates and the moving proof mass which changes the capacitance between them. However, to measure the acceleration, the transfer of charge is amplified electronically by mixing in a high frequency AC signal using a double sideband suppressed carrier modulation technique.

Finally, the amplitude-modulated signal is synchronously demodulated and amplified. The model based on the work by Grigorie [23] includes three main blocks as in Fig. 2 . There are several ways to measure the movement due to the capacitance changes. Early methods used closed loop feedback by impressing a voltage on the capacitor plates so the proof mass stays at its original position [22]. Changes in this voltage thus reflects the change in position. More recent methods are open loop, where the capacitance is obtained using switchedcapacitor techniques.

We have mentioned in Section I about the widespread use of accelerometers, but the limitations of current devices prevent them from being used in even more applications. A prime example is that of deriving the speed and distance moved by performing mathematical integration of the measured acceleration. In practice, the measurements drift and the integration causes these errors to accumulate, rendering the readings to be useless. To overcome this, it is necessary to periodically recalibrate the accelerometer readings. One popular method is zero velocity update (ZUPT), and also other schemes which depend on when an external event indicates an instantaneous null in a movement, for example in between footsteps [24].

Another source of accelerometer noise arises from mechanical sources due to the proof mass being subjected to Brownian movement. However, a lot more noise comes from electronic sources. The conversion of minute capacitance changes to usable voltages requires high electronic gain and with that, an increase in noise. A widely used method benefits from switched capacitor techniques and the oscillation frequency can feed through the main signal, resulting in aliasing effects.

This may be worsened with the use of chopper amplification with synchronous demodulation, to moderate the effect of drift.

\section{B. Practical considerations in using accelerometers}

In Section I we mentioned that accelerometers have gained even wider use by being part of consumer devices like smartphones and tablets. It is useful to differentiate between such installed units and those available as standalone units, where the accelerometer integrated circuit has settings for force sensitivity and often external components perform simple filtering of the operating frequencies. These units are small in size, consume little current but needs proper packaging to use.

By way of contrast, in ready to use consumer devices, there is no control over how an accelerometer is mounted in its physical environment. This affects its operating conditions in terms of temperature, humidity and electronic interference. In addition, further electronic filtering may be employed and the operating software can further filter the signal and introduce further latencies in signal processing. Studies concerning consumer devices may be almost immediately invalidated as they are obsoleted by the time publication takes place, as borne by new consumer models appearing every so often, with new hardware and operating systems. In these situations, in order to achieve device independence [25], there is little the user can do but to treat the accelerometer like a "black box", where the digital readout is assumed to be a measure of the acceleration and imperfections are treated statistically and data calibration would need to be employed for demanding applications. 


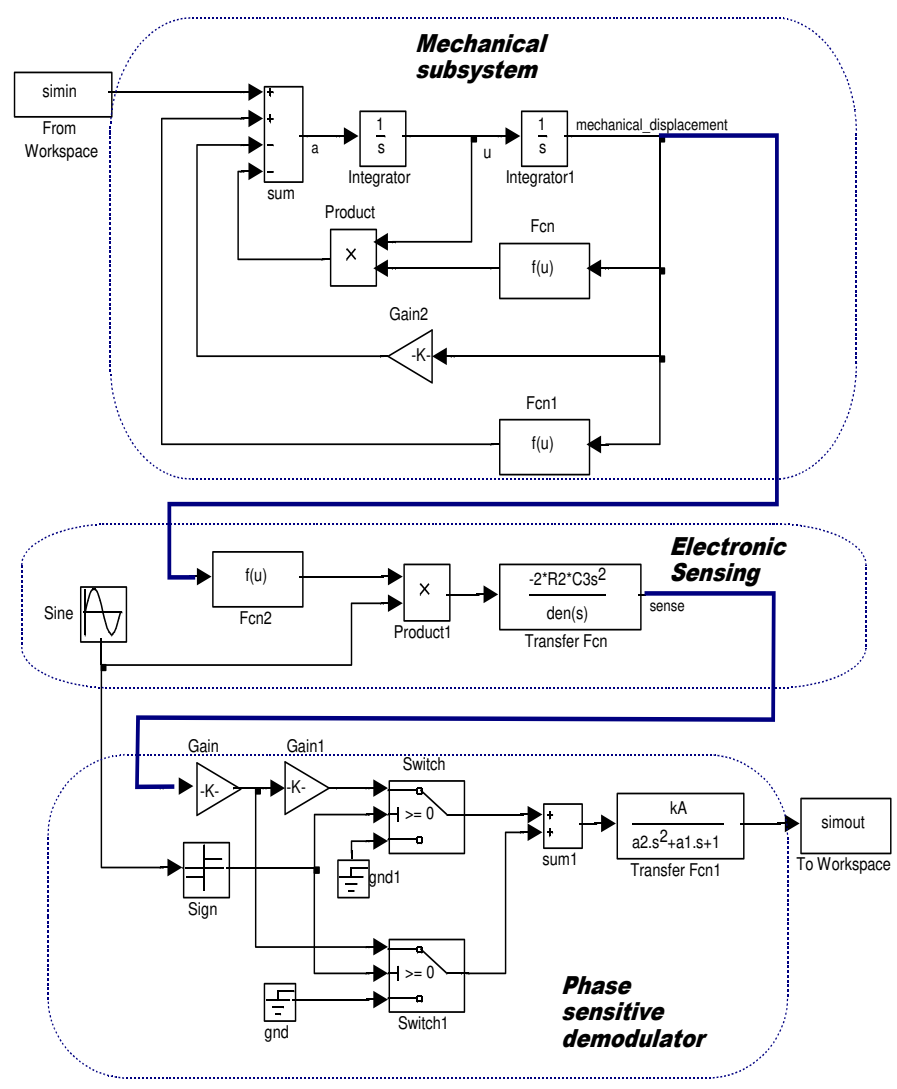

Fig. 2. Accelerometer block diagram including mechanical subsystem, electronic sensing and phase sensitive demodulator.

In this case, a model that encompasses the least common characteristics has to be used. In spite of these, the use of accelerometers in consumer devices has proven to be adequate for many clinical purposes as seen in various publications such as [26], where the measurements are compared with gold standard equipment such as motion capture cameras and accelerometers in equipment designed for clinical grade use.

\section{Other Sensing Technologies for Gait Analysis}

In this section, the selected wearable technologies used in gait analysis are briefly described. Section II has introduced the theory behind the accelerometer and its usage. The use of accelerometry for gait analysis has been significantly increased due to their ease of use, portability and the capability to be integrated into low-powered wireless embedded platforms. The aim of this section is to provide a brief overview of other popular existing wearable technologies for gait analysis.

\section{A. Gyroscope and IMU}

Of the other sensors that are used in clinical motion studies, the gyroscope is arguably the next most commonly used after accelerometer. Some important details are provided here. The gyroscope can be attached to the feet to measure the angular velocity of foot for detecting different gait phases [27]. The MEMS technology used in accelerometers is used to drive down the cost of gyroscopes so they are often featured alongside accelerometers in many devices and in IMUs. In contrast to accelerometers which can directly measure linear acceleration, gyroscopes measure the angular movement about a given axis. Accelerometers by themselves can measure angular rotation but they cannot give a good a result as gyroscopes as shown in [28]. Thus the gyroscope can be used to correct or even fused with accelerometer readings, when deployed together such as in an IMU.

There are a slew of technologies that cater to high end applications down to those in consumer devices, where the main technologies driving miniaturization are fiber optic and MEMS. Except for gyroscopes working on optical principles, what the other types have in common is a mass that is constantly moving within the device in order to measure the angular motion. This motion causes the gyroscope to consume more current than accelerometers. We examine one MEMS gyroscope technology which is based on Coriolis acceleration [29]. As opposed to centrifugal acceleration which is always present in rotation, Coriolis acceleration occurs whenever there is motion along the radius of the rotation.

Fig. 3 shows the schematic of the single axis of a gyroscope which rotates clockwise, together with its semiconductor substrate: this has capacitive fingers fabricated as part of its structure. To this substrate a frame with a set of capacitive fingers, is tethered with springs. To this frame, tethered with springs as well, is a mass driven into mechanical resonance and constrained to move in one direction. On the left of the figure, we consider the mass as it moves to the top of the figure along the radius of rotation. The Coriolis force acts on the frame which deflects to the left as shown. On the right of the figure, as the mass resonates it now moves to the bottom of the figure and now the Coriolis force causes a deflection to the right. The varying distance between the capacitive fingers is picked up as a voltage which gives a measure of the angular speed. From [28], it can be shown that the displacement of the frame relative to the substrate is:

$$
\mathbf{D}=2(\boldsymbol{\Omega} \times \mathbf{v}) M / k
$$

where $\Omega$ is the angular velocity of the gyroscope, (direction out of the page), and $\mathbf{v}$ the velocity of the resonating mass along the radius of rotation, $M$ is mass, $k$ is the spring constant of the frame-substrate springs. By driving the resonating mass at a known frequency, its changing displacement $\mathbf{D}$ induces a voltage from which the angular speed can be derived with good linearity. However, as with MEMS devices, the problems of drift and noise and other artefacts remain. An IMU can be a combination of both accelerometers and gyroscopes that measures rate, angle and direction of motion. IMUs can be attached to any part of the body and are effectively used in gait analysis. Recent IMUs have built-in magnetometers as well that can be used in fall detection.

\section{B. Force plates}

The force-plates measure vertical ground reaction forces (GRF) applied during gait by a subject. A force platform can be integrated under the moving belt of the treadmill or 


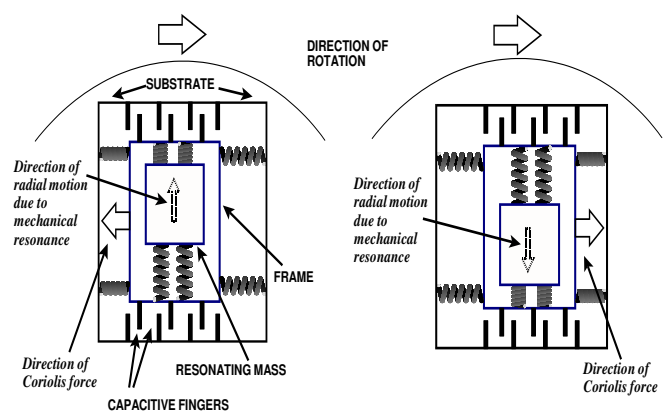

Fig. 3. Schematic of a gyroscope rotating clockwise. On left, resonating mass is moving to top of figure. On right, mass is moving to bottom of figure. Direction of Coriolis force is also shown.

under the entire treadmill [30]. Force-platforms are expensive and have some limitations such as short measurement distance and stationary property of the force plates which make them difficult to be used in free-living environments.

\section{Goniometer}

Goniometer has been used for angle measurement in gait analysis since it is flexible and can rotate proportionally to the joint angle being measured [31]. It is particularly useful for analysis of ranges of motion. Using a goniometer it is possible to determine the range of knee joint angular movement to monitor patients with knee injury. An optical fiber based goniometer has been introduced in [32].

\section{Electromyography}

Electromyography (EMG) activity can be recorded using surface electrodes or by wires inserted into the selected muscle of a lower extremity [33]. Understanding of muscle activity in gait can be obtained by analysing the EMG signals. EMG provides feasibility of analysing relative contribution of the superficial muscles during movement. It records the activity of underlying motor units and plays an important role in clinical gait analysis for assessing the walking performance of people with gait impairments using muscle activity information such as timing of the muscle activity and muscle strength.

\section{E. Sensing Fabric}

The goal in the sensing fabric based technology is integration of sensing, communication components, and the processing elements into the fabric. The most common types are pressure sensors including piezoelectric, piezoresistive, resistive, and capacitive sensors [34]. The pressure sensor based device which does fit completely inside the shoe with the capability of wireless communication is very demanding for long term monitoring and recordings of daily activities. Therefore, to make a valuable gait analysis platform, the sensing fabric technology has been directed towards the development of pressure-sensitive foot insoles with wireless communication capability [35].

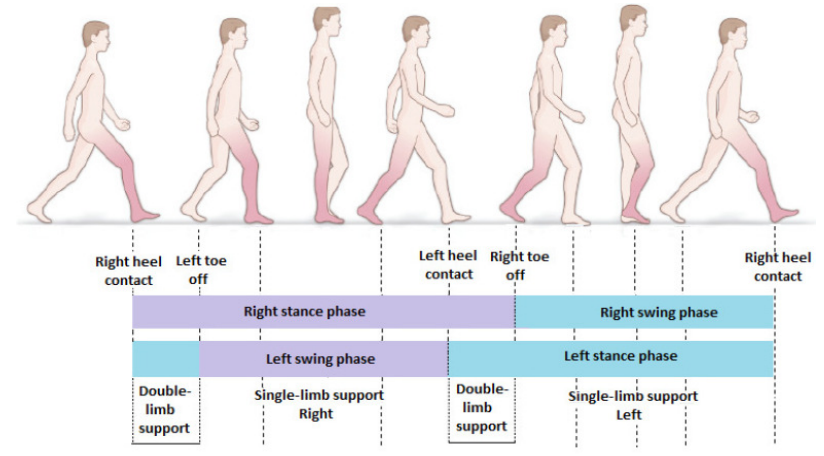

Fig. 4. The essential gait events and the corresponding temporal parameters which could be estimated using accelerometers.

In addition to the sensors involved, another factor to consider is the wireless communication capability of the wearable device. Classic Bluetooth, and Bluetooth Low Energy (BLE), are typically used along with other wireless personal area networking technologies [36], [37], such as Zigbee [38]. These commonly operate in the unlicensed $2.4 \mathrm{GHz}$ band.

Two critical issues to consider regarding wireless communication are power consumption and data rate. Trade offs must be considered between the reduced charging times and the time to download larger amounts of collected data. For example, BLE is preferred when the power consumption is more important [39] and Classic Bluetooth when the data rate is more important. If the sensed data can only be acquired via the wireless link, then, careful consideration regarding sensor sampling rates must be considered so that it does not exceed the link's capacity. In addition to a wireless link, some devices also provide a direct wired link (e.g. over a USB interface) for faster downloads [37].

\section{GAIT PHASES AND PARAMETERS}

The objective of a gait analyser system is to estimate the gait parameters as accurately as possible. In this section, the main gait phases and various gait parameters commonly used in accelerometer-based studies are explained. For some of the gait parameters, there are minor differences in their definitions.

\section{A. Gait Phases}

Each gait cycle or stride has two phases: Stance Phase, the phase during which the foot remains in contact with the ground, and the Swing Phase, the phase in which the foot is not in touch with the ground. The gait phases for each of them include [40]:

- Stance phase: initial contact, loading response, midstance, terminal stance and preswing

- Swing phase: initial swing, mid swing and terminal swing

Initial contact: the moment that the foot contacts the ground; it is also called as heel strike.

Loading response: this phase begins immediately following the initial contact of the foot and continues until the lift of contralateral limb for swing phase.

Mid stance: time period starts from the lift of the contralateral 


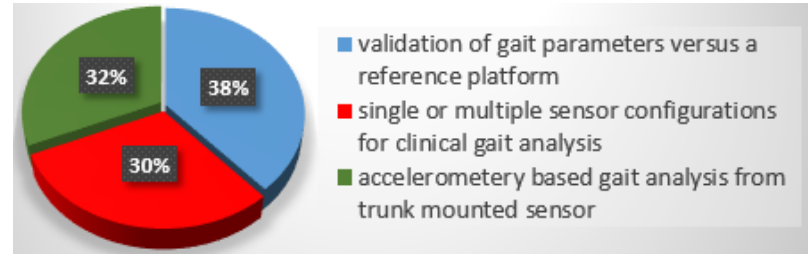

Fig. 5. 61 papers include accelerometry based gait analysis using a reference platform for validation. 48 papers include single/multiple sensor configurations mainly analysing a target clinical application excluding trunk accelerometery based gait analysis (50 papers). The papers were retrieved since 2000.

limb from the ground to the point where the body weight is aligned with the forefoot.

Terminal stance: this Period starts after heel rising in the frontal plane and continues to prior of the initial contact of the contralateral limb.

Pre swing: this phase starts from initial contact of the contralateral limb and ends with the lift of the ipsilateral limb from the ground.

Swing phase:

Initial swing: this phase, also called as toe off, is from lifting the foot off the ground until the knee flexion is increased to its maximum position.

Mid swing: this phase begins immediately after knee flexion and ends when the tibia is vertical.

Terminal swing: this phase begins following the vertical tibia position to just before the initial contact.

\section{B. Gait Parameters}

The gait parameters are obtained using the information given by the gait events identified from the output sensor signals. In Fig. 4 the essential gait parameters and gait events are provided. In the following section the spatiotemporal gait parameters are listed and explained. All listed temporal gait parameters could be estimated using the accelerometers. Of listed parameters for spatial parameters such as: step length, step width and lateral foot position, only step length has been estimated using trunk mounted accelerometers in research studies as explained in Section V-A-1.

- Acceleration amplitude variability: averaged standard deviation (SD) of the acceleration signals segmented as steps to represent the mean variability of the signal during the walking period. The steps are formed by using the acceleration peaks in the vertical axis of the acceleration signals. They are averaged and normalised by time to estimate the mean $\pm S D$. Then, the SDs are averaged to produce mean amplitude variability [41].

- Cadence: number of steps per minute (i.e. steps/min) [42][43] or number of completed steps during walking time (number of steps/walking time) [44] or number of strides per minutes [45]. The formula to derive the cadence is explained in [46] as:

$$
c=60(N / n) /(N / f)=60 f / n
$$

where $N$ is the number of samples for $D$ meter walk ( $D$ is the completed distance in meters), $f$ is the sampling frequency in $\mathrm{Hz}, n$ is the number of samples per dominant period, and $c$ is the cadence (steps/min). Having the values for $D, f, n, S$ (the time in seconds to walk $D$ meters) and $n$, it is possible to estimate other parameters, e.g. $N=S f$, $M=N / n$ ( $M$ is the number of steps per $D$ meters). Therefore, the main equation to estimate the cadence is $c=60 \mathrm{M} / \mathrm{S}$ and as seen in equation (3) it can be expressed using only two parameters $f$ and $n$ [46].

- Cycle frequency: the fundamental frequency derived by using discrete Fourier transform (DFT), since the stabilized walking generates periodic patterns in the acceleration signals [47].

- Double support duration: the duration of the phase of support on both feet. This can be also expressed as percentage of gait cycle during which both feet are in contact with the floor [48].

- Foot symmetry: the step duration expressed as percentage of gait cycle [49].

- Gait cycle time: time duration between two successive heel strike events.

- Gait irregularity: the average SDs of the left and right step times [3][50]. It shows the variability in successive steps of the same foot.

- Gait variability: the SD of gait parameters or their coefficient of variation (CV) i.e. $\mathrm{CV}=\mathrm{SD} /$ mean [51][44] which is based on stride to stride fluctuations.

- Harmonic ratio: the ratio between the sum of even and sum of odd $((\Sigma$ even harmonic $) /(\Sigma$ odd harmonic $)$ ) (for anterior-posterior and vertical axes) or the sum of odd and sum of even $((\Sigma$ odd harmonic $) /(\Sigma$ even harmonic $))$ (for mediolateral) harmonic amplitudes calculated using DFT [41]. This parameter is a representation of the smoothness and rhythmicity of acceleration patterns. The even harmonics for the AP and VT axes correspond to the inphase components of the acceleration signal while the odd harmonics indicate the out-of-phase components that are minimum for a normal gait [41].

- Interstride acceleration variability: it is measured by the autocorrelation coefficients for each axes [45].

- Lateral foot position: the distance between the heel position and its orthogonal projection from the same foot on the walking path [52].

- Normalized speed: the speed normalized w.r.t the subject's height [49].

- Root mean square: root mean square (RMS) of the acceleration magnitudes [41]. This parameter is a measure of dispersion of the accelerometry data relative to zero, in contrast to the SD which is a measure of dispersion relative to the mean of the accelerometry data. If the mean of acceleration signals is zero, the RMS has the same concept as SD. This parameter is used to indicate the average magnitude of the accelerations for each axis [41].

- Stance duration: the time from heel strike to toe off of the same foot that can also be as percentage of gait cycle.

- Step asymmetry: the ratio of the difference between mean step time of individual legs to the combined mean step time of both feet [53][50] or the difference in successive step time [3] or $A_{d 1} / A_{d 2}$ where $A_{d 1}$ and $A_{d 2}$ are the autocorrelation 
coefficients at first dominant period and second dominant period of acceleration signal (vertical or anterior-posterior) respectively [46].

- Step duration: the time between ipsilateral and contralateral heel strikes (heel contacts of the opposite foot) [49].

- Step frequency: half of the fundamental frequency calculated using DFT. It can be scaled w.r.t the subject's height to consider the influence of the height on step frequency and make a comparison for the men and women populations [54].

- Step length: covered distance in meters divided by the number of completed steps (i.e. distance $(\mathrm{m}) /$ number of completed steps) [41]. The distance between the ipsilateral and contralateral heel strikes [49]. In the most common way having the trunk accelerations, the step length can be estimated using the inverted pendulum (IP) model of walking [55][56][57][49] as:

$$
2 \sqrt{2 l h-h^{2}}
$$

where $l$ is the subject's leg length in meter $(m)$ and $h$ is the amplitude of vertical displacement during a step cycle $(m)$.

- Step regularity: $A_{d 1}$ autocorrelation coefficient at first dominant period of the vertical or anterior-posterior accelerations [46]. A higher value for $A_{d 1}$ represent higher step regularity. This is based on a fact that a periodic signal like acceleration signals generate autocorrelation coefficients which have peak values for the lags corresponding to the periodicity of the signal called the dominant period [46].

- Step timing variability: SD of time between successive heel contacts in the whole duration of walking [43].

- Step width: mediolateral distance between the heels in double support phase [58]. It was registered from footprints in [43].

- Step width variability: SD of step width [43].

- Single support duration: duration of the phase of support on one feet where can be also expressed as percentage of gait cycle during which only one feet is in contact with the floor.

- Stride duration: the time between two consecutive heel strikes of the same foot.

- Stride frequency: number of cycles per second $(\mathrm{Hz})$.

- Stride length: the distance between two consecutive heel strikes of the same foot or (average of speed $(\mathrm{m} / \mathrm{s})) / \mathrm{stride}$ frequency (in $\mathrm{Hz}$ ) [59][47] that can be scaled for the subject's height [54].

- Stride regularity: $A_{d 2}$ autocorrelation coefficient at second dominant period of the vertical or anterior-posterior accelerations [46].

- Stride symmetry: $A_{d 1} / A_{d 2} * 100$ [60][46].

- Stride velocity: the mean value of foot velocity in forward direction during gait cycle [51], or ratio of the stride length to stride time [48].

- Swing duration: the time from toe-off to heel strike of the same foot that can also be expressed as percentage of gait cycle.

- Walking distance: multiplication of mean step length over a specified duration by the number of steps.
- Walking intensity: calculated from the integral of the modulus accelerometer output [45].

- Walk ratio: the average step length (in $\mathrm{cm}$ ) divided by cadence (steps/min)[41]. This parameter indicates the association between the amplitude and frequency of the rhythmic foot movements during walking [41].

- Walking (gait) speed: measured in meters per second using a chronometer [47] or having distance divided by the walking time [44].

- Walking time: measured using stop watch as in [57].

- Walking velocity: \{distance covered/number of data points/sampling frequency\} [41] which results in meters per second.

Depending on the sensor location, experiments (level walking, walking on treadmill or irregular surfaces, etc) and also testing on healthy subjects or patients with pathological gait, various errors versus the reference platform are reported in the literature. The accuracies of temporal gait parameters or events are measured and compared with the reference platform typically in terms of seconds or milliseconds. For spatial parameters such as step or stride length, the accuracies are described in meters. Some other parameters are described as a ratio such as stride/step asymmetry to compare normal and asymmetric gait. Usually, a perfect step symmetry has a value close to one (or 100\%) and completely asymmetrical walking is represented as zero (or $0 \%$ ). As an example, step asymmetry has been measured as 0.98 and 0.31 for normal and asymmetric gait respectively, in [46]. In another study [60], the reproducibility of the gait measurements are analysed using intraclass correlation coefficients (ICCs) where a high degree of reliability (e.g. the maximum reliability is presented as one) with repeatability of 0.72 and 0.93 have been obtained for both stride regularity and stride symmetry, respectively. In the following section detailed explanations for validations using reference platforms and reliability assessments are provided.

\section{ACCELEROMETRY BASED GAIT ANALYSiS}

Three databases including PubMed, Web of Science and MEDLINE were selected. The search string was created as:

(gait OR walking) AND (acceleration OR accelerometer OR accelerometry) AND (heel strike OR toe off OR push off OR swing OR stance OR cycle OR parameter OR initial contact OR terminal contact)

The above terms were used to search the title and abstract of the articles. After retrieving the articles, first the duplicates were removed. Then, the articles from 2000 onwards were selected for screening. The total of 994 articles were screened in two stages. In the first stage the titles and in the second stage the abstracts of the articles were reviewed. A total of 159 articles were selected for full review. These papers are divided in three groups as outlined in Fig. 5. The papers include single or multiple accelerometry based systems which are used mainly for validation of gait parameters/event versus a reference platform (section V-A). These papers could also include a target clinical application. Then, the papers which mainly include a target clinical application with trivial or no 
validation studies (section V-B), excluding trunk accelerometry based analysis, are presented. The papers including trunk accelerometry with a clinical application are described in section V-C.

\section{A. Platforms for validation}

Development of wearable accelerometry based gait analysis systems requires a systematic validation using the established techniques. Most of these techniques are limited to gait analysis laboratories that include expensive devices such as instrumented walkway also limiting the number of strides. These devices do not necessarily reflect the gait dynamics while walking in non-laboratory environments.

On the other hand, force sensitive resistors or footswitches can be used to evaluate the accuracy of an accelerometery based gait analysis systems. These devices can be used for outside laboratory gait validation.

The most commonly used platforms include video images, force-plate measurements and pressure sensing platforms to validate the accuracy of the estimated gait parameters or events. Video images have been used in [57][61][62][63][64][65] for validation purposes. More specifically, the used platforms are VICON Motion Capture, Oxford, UK [66],[67], Stereophotogrammetric unit with eight M2-cameras (VICON) and two dynamometric platforms [49], Cartesian Optoelectronic Dynamic Anthropometer (CODA) motion analysis system [68][69][70]. Other systems include Optical Motion Capture Systems (OMCS) [71][72][73], optical position measurement system (Optotrak) [52], footswitch or Force Sensing Resistors (FSR) [74][75][76][77][78][79], foot plantar pressure system [80], GAITRite [53][44][81][82], and force-plate measurements [83][84][85][86][87][88][89][56][90][91][65][92]. Microsoft (MS) Kinect image and depth sensors provide a simple 3D modeling using image and depth sensors which have shown to be effective in analysis of gait disorders [93] and to recognise PD gait [94].

Another form of validating the estimated gait parameters is test-retest reliability assessments. Based on this scheme using repetitive experiments, the trials are performed in different conditions or sessions. Then, the intra-session (using the trials within sessions) and inter-session (using trials between sessions) reliability of gait variables are evaluated using the ICCs as an indication of relative reliability and $\mathrm{CV}$ for absolute reliability [95][81]. There are six universal forms of the ICCs which may produce different results [96]. Therefore, it is necessary to correctly interpret them for a reliability study in the context of gait analysis. A brief general guideline for selection of ICCs is provided in the following. Suppose there are $n$ subjects $(j=1, \ldots, n)$ in a study, then $k$ tests $(i=1, \ldots, k)$, are performed for each subject. Let $y_{i j}$ be a recorded quantity (it can represent an estimated gait parameter) for the $j^{\text {th }}$ subject in the $i^{\text {th }}$ test/measurement using the unified ANOVA model:

$$
y_{i j}=\mu+b_{j}+a_{i}+(a b)_{i j}+e_{i j}
$$

where $\mu$ is the overall population mean of measurements, $b_{j}$ is the deviation from the mean of the $j^{\text {th }}$ subject's measurements (i.e. mean across repeated measurements on $j^{\text {th }}$ subject), $a_{i}$ is the difference from mean of $i^{t h}$ test and $(a b)_{i j}$ is the difference of repeated measurements of $j^{\text {th }}$ subject in $i^{t h}$ test, $e_{i j}$ is the random error in the measurement of $j^{\text {th }}$ subject in the $i^{\text {th }}$ test. This model relies on the fact that the measurement reflects a combination of true condition of the subject (i.e. $\mu+b_{j}$ ) and measurement errors $\left(a_{i}+(a b)_{i j}+e_{i j}\right)$. In the absence of repeated measurements in the $i^{\text {th }}$ test $(a b)_{i j}=0$.

1) One-way random effect model $y_{i j}=\mu+b_{j}+e_{i j}$, systematic error of test is insignificant

2) Two-way random effect model $y_{i j}=\mu+b_{j}+a_{i}+(a b)_{i j}+$ $e_{i j}, a_{i} \sim \mathbf{N}\left(0, \sigma_{J}^{2}\right),(a b)_{i j} \sim \mathbf{N}\left(0, \sigma_{I}^{2}\right)$

3) Two-way mixed effect model $y_{i j}=\mu+b_{j}+a_{i}+(a b)_{i j}+e_{i j}$, $\sum_{i=1}^{k} a_{i}=0, \sum_{i=1}^{k}(a b)_{i j}=0$

If the systematic error $\left((a b)_{i j}+a_{i}\right)$ is negligible or the errors in the repeated tests do not differ significantly, model (1) can be used. Also, if different devices are used in the tests, or the tests are conducted at different locations or time slots, and the effects of test/measurement and subjects are inseparable, model (1) should be used. This can also be determined using F-test (e.g. the ratio of between-subjects to within-subjects mean squares) and evaluating the resulted p-value to find the significance of systematic errors. If the systematic error is significant, model (2) or (3) should be used. In random effect model (2) the systematic errors in all possible tests are random and do not have any pattern (e.g., the systematic error in later tests is smaller or larger than that in previous tests). In other words, the results from the particular $k$ tests conducted in this study can be generalized to all possible tests. In contrast, test is treated as a fixed factor model (3), in the two-way mixedeffect model. This means that the results from the conducted tests are not random and, thus, cannot be generalized to all possible tests or such a generalization is not of interest. ICCs can be calculated as:

$$
\begin{aligned}
I C C(1,1) & =\frac{B M S-W M S}{B M S+(k-1) W M S}, I C C(1, k)=\frac{B M S-W M S}{B M S} \\
I C C(2,1) & =\frac{B M S-E M S}{B M S+(k-1) E M S+k(J M S-E M S) / n} \\
I C C(2, k) & =\frac{B M S-E M S}{B M S+(J M S-E M S) / n} \\
I C C(3,1) & =\frac{B M S-E M S}{B M S+(k-1) E M S}, I C C(3, k)=\frac{B M S-E M S}{B M S}
\end{aligned}
$$

$B M S$ is between-subjects mean squares, $W M S$ is withinsubject mean squares, $E M S$ residual mean squares and $J M S$ is between tests's mean squares. where the first index in ICC indicates one of the three underlying ANOVA models, and the second index indicates whether the reliability of a single measurement $(k=1)$ or that of the average measurement (over $k$ repeated tests, $k=2$ for a test-retest case) is considered. $I C C(1,1) / I C C(1, k)$ and $\operatorname{ICC}(2,1) / I C C(2, k)$ are measures of test agreement as the between-tests variance is included in their denominators. $\operatorname{ICC}(2,1) / \operatorname{ICC}(2, k)$ are usually used to generalize the results in a study to all possible tests. $\operatorname{ICC}(3,1) / \operatorname{ICC}(3, k)$ are measures of test consistency as the between-tests variance is not included in their denominators and are useful when the relative differences in the test are required. ICCs can be used to decide the number 
of trials for a reliable inference. As an example, suppose a measurement should be repeated for estimation of a selected gait parameter. Then, after two trials, after including the third trials, if re-calculated ICC increases, it means adding a third trial can enhance the intertest reliability.

The reliability of DynaPort (a GaitMonitor software for estimating the gait parameters using a single trunk accelerometer device) has been tested in [44] for estimation of gait parameters related to heel strikes in older adults in which the system has been validated using the GAITRite system (CIR Systems Inc., PA, USA). $\operatorname{ICC}(2, k)$ has been used to find the levels of agreement between Dynaport and GAITRite systems [44]. $\operatorname{ICC}(2, k)$, with $k=4$, has been used to assess validity of averaged gait parameters over four walking trials. $I C C(2,1)$ has been used for evaluation of individual footsteps from step duration and step length. Given the gait limitation using GAITRite system on a fixed and flat surface, the reliability of spatiotemporal gait parameters using different walking conditions and considering dual task conditions in older adult population using DynaPort system has been assessed in [81]. The reliabilities of $\operatorname{ICC}(2,1)$ and $\operatorname{ICC}(3,1)$ have been used to express interrater and intrarater reliabilities respectively. Validation of the CODA system for gait measurements has been addressed in [133] using intrarater and interrater reliability assessments from $\operatorname{ICC}(2,1)$ emphasizing on generalisability from two groups of subjects.

IDEEA (MiniSun, Fresno, CA) is an accelerometry based gait analysis system which can be used for other purposes such as physical activity assessment and estimation of energy expenditure. This system consists of five biaxial accelerometers where the spatiotemporal gait parameters are estimated. The validity of this system is compared to forceplate measurements in [86]. This system has been also used for determination of gait characteristics in older adults [134].

In [86], intrasession reliability of IDEEA was shown to be excellent. However, walking speed was significantly underestimated compared with force plate-based analyses. Concurrent validity of the IDEEA system versus forceplates is performed using $\operatorname{ICC}(2,1)$. Intrasession reliability of each system has been expressed separately using $\operatorname{ICC}(3,1)$.

In [75] six state of the art methods are compared in terms of accuracy of the gait events versus the reference data using MAREA database. Supervised machine learning has been used for gait event detection during the application of functional electrical stimulation (FES) using a single shank mounted accelerometer [74]. The lumbar and shank accelerometers were found as the most accurate alternatives to the shank gyroscope for gait event detection and temporal parameters assessment, respectively [92]. In [135] two triaxial accelerometers placed on the left and right posterior superior iliac spine have been used to detect gait phases in healthy subjects and free living environment to propose an alternative method for trunk acceleration studies. Reliability of Rehawatch for estimating spatio-temporal gait parameters has been evaluated [136]. In [137], an iPhone has been used to estimate gait cycle, step cycle time and step duration cycle.

The reliability of spatiotemporal gait parameters in single and $k$ repeated measurements $(k=3)$ have been assessed using $\operatorname{ICC}(2,1)$ and $\operatorname{ICC}(2,3)$ for IMU-based sensor and camera systems [138]. The reliability of inertial senors has been quantified using $\operatorname{ICC}(2,1)$ for each condition (varied incline and speed) using RehaGait and instrumented treadmill [139][140]. In [141], $\operatorname{ICC}(1,1)$ has been used as reliability measure of gait variability in older adults. In [142], $\operatorname{ICC}(2, k)$ is used to determine the reliability of gait variables during preferred and fast walking conditions. In [143] test-retest reliability assessment of gait variables in older adults with mild cognitive impairment (MCI) under single and dual-task conditions has been performed using $\operatorname{ICC}(2,1)$.

In [144], the reliability of gait parameters has been assessed using $I C C(2,1)$ and $I C C(1,1)$ for analysis of acceleometer and IPod Touch which is disputable. In a recent work [145], the $\operatorname{ICC}(2, \mathrm{k})$ form has been used for freezing time percentage and number of FoG events for feature selection.

1) Step length/walking speed estimation: An artificial neural network (ANN) has been used in [146] to estimate the walking speed using a single triaxial accelerometer placed on the upper thigh. For this research, the input to the ANN includes six parameters e.g. step number, subject's height, root mean square and difference between the maximum and minimum of vertical and frontal accelerometer signals. In [147] a single shank mounted IMU has been used to estimate the stride length, walking speed and slope estimation taking advantage of the IP model where the speed validation has been performed by the use of treadmill. The velocity and displacement are estimated using single and double integration of the accelerations in [49]. Then, all the spatio-temporal gait parameters are calculated using only acceleration signals recorded using an IMU placed on the lower trunk. Parameters such as speed, stride time, and stride length are estimated from chest accelerometer [148] during treadmill exercise test with the help of an ANN-based subject-independent algorithm.

In [62], the inverted pendulum equation has been used to estimate the walking distance and step length from a single accelerometer placed on the lower trunk. Based on the results, the hypothesis that the IP model accounts for the displacement during single stance phase has been supported. In [149] the inverted pendulum-like behavior of the stance leg during walking has been used to identify individual stride cycles using the direct integration of the accelerometer signals. It shows that two-shank mounted accelerometers are useful to estimate walking speed which are validated using treadmill information.

The reliability and validity of four different IP based models for estimation of mean step length in independent-living have been assessed using old subjects [150]. For all step-length estimators, the test-retest intra-class correlations approached or were above 0.90. Agreement of each IP based model of step length with reference has been evaluated using $\operatorname{ICC}(3,1)$. The reliability of step length between session trials which include different speeds and conditions (dual task) has been assessed using $\operatorname{ICC}(2,1)$. An unbiased autocorrelation procedure has been used to estimate the cadence, step length, gait regularity (step, stride) and step symmetry (vertical and mediolateral) using trunk mounted accelerometer [46]. Speeddependent changes in acceleration are investigated based on two running trials on a treadmill and on land at three speeds 
TABLE I

SINGLE OR MULTIPLE SENSOR CONFIGURATIONS WITH CORRESPONDING APPLICATIONS

\begin{tabular}{|c|c|c|c|c|}
\hline $\begin{array}{l}\text { sensor } \\
\text { number }\end{array}$ & sensor position & sensor type & application & reference \\
\hline 㟆 & $\begin{array}{l}\text { - } \text { Ear } \\
\text { - Ear } \\
\text { - Ear } \\
\text { - person's prosthesis } \\
\text { - thorax } \\
\text { - } \text { ghoe (distal end) } \\
\text { greater trochanter } \\
\text { - } \text { heenk } \\
\text { - knee } \\
\text { - thigh } \\
\text { - thorax } \\
\text { - right ankle } \\
\text { - } \text { shank } \\
\text { - right front hoof } \\
\end{array}$ & $\begin{array}{l}\text { - triaxial accelerometer } \\
\text { - triaxial accelerometer } \\
\text { - triaxial accelerometer } \\
\text { - biaxial-uniaxial accelerometers } \\
\text { - triaxial accelerometer } \\
\text { - } \text { tri-axial accelerometer } \\
\text { - tri-axial accelerometer } \\
\text { - dual-axis accelerometer } \\
\text { - } \text { bi-axial accelerometer } \\
\text { - IMU accelerometer } \\
\text { - } \text { tri-axial accelemeter } \\
\text { - } \text { IMU } \\
\text { - tri-axial accelerometer } \\
\text { trial accelerometer } \\
\end{array}$ & $\begin{array}{l}\text { - To evaluate Gait changes of PD patients after modification of DBS parameter } \\
\text { - To monitor rehabilitation of patients following lower limb reconstruction } \\
\text { - To detect gait asymmetry in older adults including orthopaedic patients } \\
\text { - To assess walking pattern of patients with a lower-limb amputation } \\
\text { - To differentiate between pre-manifest and manifest Huntington's disease subjects } \\
\text { - To monitor vertical toe clearance during walking } \\
\text { - To evaluate the dynamic instability of the dysplastic hip } \\
\text { - To detect the gait phases in normal and hemiplegic gaits } \\
\text { - To detect IC and TC ing in stride from normalthy subjects and trans in ITW children } \\
\text { - To detect the swing phase of stroke gait in functional electrical stimulation } \\
\text { - To differentiate between amputee and control } \\
\text { To investigate relationship of immersion depth and impact loading to diminish injury risk } \\
\text { - To real-time detection of gait events for lower limb amputees } \\
\text { - To identify gait differences between single-task and dual-task walking in older individuals } \\
\end{array}$ & $\begin{array}{l}{[97]} \\
{[98],[99]} \\
{[100]} \\
{[61]} \\
{[53]} \\
{[101]} \\
{[102]} \\
{[103]} \\
{[104]} \\
{[88]} \\
{[105]} \\
{[106]} \\
{[107]} \\
{[108]} \\
{[109]} \\
{[110]}\end{array}$ \\
\hline$\stackrel{0}{2}$ & $\begin{array}{ll} & \text { shoe (right-left) } \\
& \\
\text { - } & \text { forehead-sternum } \\
\text { - } & \text { shank-trunk } \\
\text { - } & \text { ankle (right-left) } \\
\text { - foot-shank } \\
\text { - head-trunk } \\
\text { - head-sacrum } \\
\text { - head-pelvice } \\
\text { : head-pelvice } \\
\text { - } \text { head-pelvice } \\
\text { - knee-ankle head } \\
\text { - right hip - lower back } \\
\text { - hind leg (left-right) } \\
\text { - shoe (left-right) } \\
\text { - shoe (left-right) } \\
\text { - } \text { shoe (left-right) } \\
\end{array}$ & $\begin{array}{l}\text { - } \text { IMU } \\
\text { - } \text { biaxial accelerometers } \\
\text { - } \text { IMU } \\
\text { - dualaxial gyroscope-accelemeter } \\
\text { - bi-axial accelemeter } \\
\text { - tri-axial accelemeter-gyroscope } \\
\text { - tri-axial accelemeter } \\
\text { - tri-axial accelemeter } \\
\text { - tri-axial accelemeter } \\
\text { - } \text { bi-axial accelemeter } \\
\text { - tri-axial accelemeter } \\
\text { - tri-axial accelerometer } \\
\text { - } \text { IMU } \\
\text { - } \text { IMU } \\
\text { - } \text { IMU }\end{array}$ & $\begin{array}{l}\text { - To investigate gait parameter changes before and after rehabilitation - To evaluate age related } \\
\text { gait and balance performance - neuro rehabilitation } \\
\text { - To classify elderly with Orthostatic Hypotension }(\mathrm{OH}) \text {, elderly fallers without } \mathrm{OH} \text { and control } \\
\text { - To discriminate between fallers and non-fallers } \\
\text { - To investigate gait changes in moderate/mild Duchenne Muscular Dystrophy patients } \\
\text { - To discriminate gait phase in Parkisonian gait } \\
\text { - To detect IC and EC for normal and spinal-cord injured subjects } \\
\text { - To find gaiterences in young and elderly groups } \\
\text { - To evaluate walking on in PD with no history of fall, PD with a history of fall and controls } \\
\text { - To evaluate young and older subjects walking on a level and an irregular walking surface } \\
\text { - To evaluate gait in older people with diabetic peripheral neuropathy (DPN) and controls, } \\
\text { - To evaluate hemiplegic gait with different Brunnstrom stages } \\
\text { - To control an intelligent knee prosthesis for above-knee amputees } \\
\text { - To detect unilateral hind limb lameness and foot pathologies in dairy cows } \\
\text { - To automate detection of stair-climbing gait events in children for motivational therapy study } \\
\text { - To analyse the gait of geriatric patients } \\
\text { - To propose and validate an automated TUG phase classification methodology } \\
\text { To validate foot-worn IMUs for the gait measurement of children with cerebral palsy }\end{array}$ & $\begin{array}{l}{[51]} \\
{[76]} \\
{[48]} \\
{[111]} \\
{[13]} \\
{[172]} \\
{[113]} \\
{[41]} \\
{[42]} \\
{[114]} \\
{[115]} \\
{[116]} \\
{[117]} \\
{[118]} \\
{[119]} \\
{[121]} \\
{[122]} \\
\end{array}$ \\
\hline 莊 & $\begin{array}{l}\text { - lower back, thigh (right-left) } \\
\text { - shank-thigh-foot dorsum } \\
\text { - shank-thigh-foot } \\
\text { - trunk-thigh-foot } \\
\end{array}$ & $\begin{array}{ll} & \text { IMU } \\
: & \text { IMU } \\
: & \text { IMU } \\
: \text { two-3D acclerometer } & \text { IMU } \\
\end{array}$ & $\begin{array}{l}\text { - To classify normal, elderly, and elderly with support } \\
\text { - To discriminate gait symptoms between hemiplegia and asymptomatic subjects } \\
\text { To identify gait event on persons with dropped foot } \\
\text { - To examine the difference between healthy and hemiparetic gait } \\
\text { - To analyse the gait and balance in patients with Alzheimer's disease }\end{array}$ & $\begin{array}{l}{[123]} \\
{[124]} \\
{[125]} \\
{[126]} \\
{[127]}\end{array}$ \\
\hline 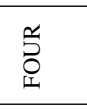 & $\begin{array}{l}\text { - metatarsophalangian joint with } \\
\text { hallux, above lateral malleolus } \\
\text { - lower leg } \\
\text { - head-lower back-foot(left-right) }\end{array}$ & $\begin{array}{l}\text { - tri-axial accelerometer } \\
\text { - } \text { tri-axial acclerometer } \\
\text { - IMU }\end{array}$ & $\begin{array}{l}\text { - To quantify gait asymmetries and FoG in PD patients } \\
\text { - To measure temporal gait characteristics in dogs } \\
\text { - To find features correlated with severity of lower limb osteoarthritis using WOMAC index }\end{array}$ & $\begin{array}{l}{[69]} \\
{[128]} \\
{[129]}\end{array}$ \\
\hline 宏 & $\begin{array}{l}\text { - above malleolus (2), on the wrist } \\
\text { dorsum(2), the upper trunk and } \\
\text { the lumbar trunk }\end{array}$ & - IMU & - To detect balance and gait deficits in people with multiple sclerosis & [131] \\
\hline $\begin{array}{c}\mathrm{Z}_{1} \\
\text { 秥 } \\
\end{array}$ & $\begin{array}{l}\text { - pelvis, thigh (left-right), } \\
\text { shank (left-right), } \\
\text { feet(left-right) }\end{array}$ & - IMU & - To characterise the gait of osteoarthritis patients & [132] \\
\hline
\end{tabular}

(slow, preferred, and fast) [151]. Parameters including amplitude and RMS of acceleration and stride duration were estimated by performing test-retest reliability assessments. It has been investigated in [152] whether an inertial sensor could discriminate between normal walking, fast walking, and running while using a treadmill for validation. The trials were performed on both overground and treadmill settings. A significant difference $(\mathrm{P}<0.001)$ in the occurrence of heel strike in the gait cycle was found between running and the two walking conditions.

In [153], an adaptive Kalman filter based sensor fusion system (considering two accelerometer axis and one gyroscope axis) has been proposed to estimate the walking distance as well as the step/stride length using sensors placed in the user shoe. The fusion of accelerometer and gyroscope axes have shown to be more accurate for estimating the spatial parameters. The signals from both accelerometers and gyroscope are used in [78] to estimate the traversed distance in level walking with varying speed where the step length has been compared using footswitches. Step length has been estimated from an IMU placed on the pelvis. It has been validated using the stereo-photogrammetric system [154] by combination of a Kalman filter and an optimally filtered IMU signals by applying direct and reverse integrations.

In [155], the walking speed estimation has been validated using both acceleration and gyroscope signals from a single shank mounted IMU. Shank mounted sensors could be very useful for walking speed estimation in abnormal foot motion which could be also used for the embedded control of kneemounted devices including energy harvesters and prostheses. Shank-mounted IMU has been used to estimate the step length [156] and walking speed [156][157]. High-speed video has been effectively used in [84] for validation of temporal gait parameters for elite athletes while a force platform has been used to validate the gait parameters for amateur subjects during sprint running.

In [158] the gait pattern of healthy old people is assessed during three clinically relevant walking conditions (self- 
selected speed (CW), fast self-selected speed (FW), and finally in dual task walking condition (DTW)) simultaneously using an accelerometer and an opto-electronic method to evaluate the gait parameters normalized to the leg length. The Codamotion system has been used to estimate swing time, stance time, right and left double support time [158]. The gait speed, stride length and stride frequency were measured by the Locometrix system.

Force plate and video images have been used for validation of stride length and stride quality from accelerometers placed on the right ankle of children [159]. The correlation between the stride quality and the body mass index has been found to be negative. An algorithm has been presented in [160] to identify the peaks corresponding to heel strike (HS) and toe off (TO) events based on scale-space filtering using Gaussian kernels. In addition, comparison of temporal gait parameters and Tinetti gait and balance average scores between the two groups of young and pre-fail older adults has been performed [160]. Real-time Detection of gait events (HS, TO) based on single accelerometer placed on the right lower leg considering different terrains has been performed using time-frequency analysis and Peak Heuristics Algorithm [161]. The walking conditions include level walking as well as upstairs and downstairs walking. It was validated against FSR on the right foot. A study in [162] investigated outdoor surface effects on dynamic stability and loading during running using tri-axial trunk accelerometry. No difference was found for concrete road and synthetic track. The results suggest that woodchip trails distort aspects of loading and dynamic stability that are detectable using a single trunk mounted accelerometer.

A system including IMU placed on the dorsum of the foot has been developed in [163] to monitor human gait in outdoor using an inertial measurement unit for early detection or prevention of falls in older adults. The system has been validated using a motion capture system. Shank-mounted gyroscopes are compared with three benchmark systems, namely accelerometers, pressure-sensitive soles and a motion capture system (Oqus camera system, Qualisys AB, and Gothenburg Sweden) in [164] to validate the HS and TO.

It has been concluded that the heel strike events correspond to a trough in the gyroscope curve, being consistent with previously reported studies. However, the toe-off event clearly did not concur with another trough of the gyroscope trace where it was established as an assumption. Regardless of the gait speed, the heel-off event was reliably at $51 \%$ of the step cycle. The findings were noted to be crucial for gait recording systems which aim to assess both the temporal and spatial aspects of human gait. Using a smartphone, the stride length has been estimated and validated using six infrared cameras [165]. A comparative analysis of methods for evaluation of the estimated initial and terminal contacts w.r.t. the number of extra and missed events as accuracy and robustness to IMU location has been performed in [166]. Validated platforms include stereo-photogrammetric system and two force platforms.

\section{B. Gait analysis for clinical applications}

As included in many of the above studies, estimation of gait parameters has been shown to be very useful for numerous clinical applications. In Table I, detailed information including the sensor type, position and clinical application for 48 selected papers from 2000 onwards are provided. The ear-worn sensor has shown consistent placement and has been used in the fracture clinics to monitor the rehabilitation of orthopaedic patients [100] and patients with lower limb reconstruction [98], [99]. It has also been used in the Parkinson's gait lab at University College London for PD gait analysis [97].

One of the most well known application is to differentiate the gait characteristics of the healthy normal subjects and those of people with walking impairments. This includes patients with pathological gait disorders such as those caused by cerebral palsy [130][122], spinal-cord injuries [112], transtibial amputation [88], lower limb amputation [61][106][108], hemiplegic gaits [124][103][126], hip dysplasia [102], Parkinson's disease (PD) [63][69], geriatric disorder [120], osteoarthritis [132] and orthopaedic [100]. It is also applied to monitor rehabilitation of patients following anterior cruciate ligament and lower limb reconstruction [98], [99].

PD gait has been evaluated in [113] to discriminate between the PD patients with or without fall history. In a recent study, the relation between the step frequency and the change in deep brain stimulation (DBS) frequency has been investigated [97]. For this novel study, the frequency of the implanted electrodes of the patients treated with DBS of the subthalamic nucleusbrain has been changed using a controller by the neurologist. Up to six different DBS frequencies $(40 \mathrm{~Hz}-140 \mathrm{~Hz}$ with $20 \mathrm{~Hz}$ intervals) have been tested while keeping the total energy constant (including 30 minutes time gap after a frequency change). The selected gait parameter from a single ear-worn accelerometer sensor has been validated versus CODA motion capture system and has shown promising results.

Gait parameters and events have been used in many clinical applications, such as to distinguish toe walking stride from normal stride in Idiopathic Toe Walking (ITW) children [104], find differences in young and elderly groups [77], classify elderly with Orthostatic Hypotension (OH), study of elderly fallers without $\mathrm{OH}$ and control [76], evaluate gait in old subjects with diabetic peripheral neuropathy (DPN) and in agematched controls [114], differentiate between pre-manifest and manifest huntington's disease [53], evaluate hemiplegic gait with different Brunnstrom stages [115], distinguish between different disease stages in children with Duchenne Muscular Dystrophy [111], evaluate walking on level and irregular surfaces [41], the same study for both young and older subjects [42], control an intelligent knee prosthesis for above-knee amputees [116], automate detection of stair-climbing gait events in children as a motivational therapy study [119], propose and validate an automated Timed up and go (TUG) phase classification methodology [121], classify normal, elderly, and elderly with support [123], identify gait events on persons with dropped foot [125], find features that correlate with the severity of lower limb osteoarthritis evaluated by the WOMAC index [129], determine the reliability of spatiotemporal gait 
TABLE II

TRUNK ACCELEROMETRY

- stride _time, walking intensity, cadence,_walking_(inter-stride) variability

- stride frequency, step symmetry, stride regularity, cranialcaudal activity, harmonic analysis

- walking speed, cycle frequency, stride length, side-to-side symmetry, step regularity, $\overline{C r}$ - $\overline{\text { raniocaudal }}-\overline{-}$

number of steps, walking distance, step length, velocity, step duration

- $\overline{\mathrm{R}} \overline{\mathrm{M}} \mathrm{S}$ from $\mathrm{AP}$ and $\bar{M} \overline{\mathrm{L}}$ axes, frequency of sway

- Gait speed, stride length, and cadence, harmonic ratio

- gait velocity, stride duration, stride length and gait regularity (ML, $-\overline{\mathrm{AP}} \overline{\mathrm{P}} \mathrm{\textrm {VT }})$

- step time, stride length and walking

- mean acceleration; mean walking speed; The ratio between the accelerations

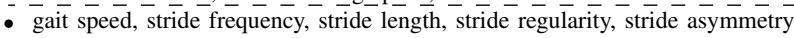

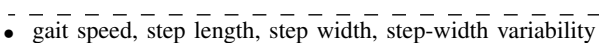

Trunk movement regularity and asymmetry

- peak evaluation of accelerations

- step count, mean step time, step length, gait speed, step time intact leg, step time prosthetic $\bar{l} \bar{l} \overline{-}-\bar{c}$

stride related measures, stride time, gait speed, mean and correlation of variation of - stride times

- acceleration $\overline{\mathrm{RM}} \overline{\mathrm{S}}$, gait harmony

- speed, stride frequency, mean stride time, $\bar{C} \overline{\mathrm{V}}$ stride $\overline{\text { time }}, \overline{\mathrm{P}} \overline{\mathrm{P}} \overline{\mathrm{I}}$, $\overline{\text { stride-to-stride }}-\overline{-}-\overline{a r i a b i l i t y}$

step time, stride time

- step events

- Speed, Stride frequency, Regularity

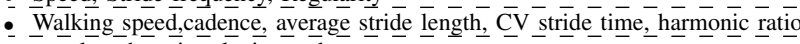

- step length, gait velocity, cadence

- Cadence, step width, step width variability, mediolateral interstride trunk autocorrelation

step regularity

- step length, speed, step time, cadence, asymmetry, irregularity, vertical displacement

- step time, step length, step frequency and walking speed, irregularity, leftright asymmetry, - displacement

- speed, step length, step duration, cadence, vertical displacement, asymmetry, and irregularity

- walking speed, frequency, step length, asymmetry, RMS (VT), Harmonic ratio (VT), inter-stride variability (VT)

- stride regularity, stride symmetry

- Max, Maxdiff, Maxp2p, Maxp2pdiff

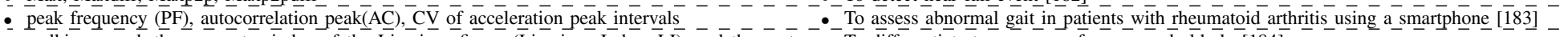

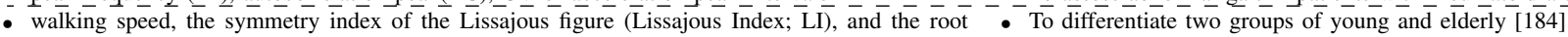
mean square (RMS) and harmonic ratio (HR) of the acceleration signals

- cadence, step regularity, stride regularity and step symme-

- step length, step frequency

- duration of subsequent stride cycles and left/right steps, step length, walking speed

- gait cycle, stance phase-

- step time, step length

- MinDiff, MaxDiff, difference between min. and max. values reached over stride cycle

- cadence (step frequency), stride regularity (peak anterior acceleration)

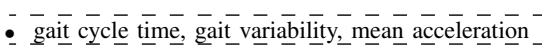

- mean step time, mean stride time, RM $\bar{M}$, stride length, gait variability

- gait cycle, peak acceleration

- gait cycle, gait velocity, $\overline{\text { stride }} \overline{\text { time, }} \overline{-} \overline{\text { step time, }} \overline{\text { stance time, }} \overline{-}-\overline{\text { swing time }}-\overline{-}$

- $\overline{\text { foot }}$ contacts, $\overline{\text { index }} \overline{-}$ of harmonicity, and amplitude variability

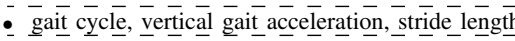

- gait speed, Lyapunov exponents, harmonic ratios, Lempel-Ziv complexity, (standard $\overline{\text { deviation, }} \overline{-}$ kurtosis, skewness) of signal amplitude, entropy rate, wavelet bands, wavelet entropy

$\because$ gait cycle, gait variability, single support, double support, step length, cadence - - - -

parameters during level walking and stair climbing [95], and detect swing phase of stroke gait during the FES process, which has the benefit of using the stimulator to correct the foot dropped gait [105].

In [95], the reliability of the IDEEA system (consists of five biaxial accelerometers) is tested for level walking and stair climbing in which the results demonstrate the highly accurate measurement of gait parameters during the level walking although with moderately less correlations for stair climbing. Another study has shown that the IDEEA monitor has lower levels of repeatability compared to three-dimensional gait analysis (3-DGA) in individuals with cerebral palsy [130] and there are systematic errors including under and over-estimation
-_To investigate COPD_patients_gait patterns versus healthy_subjects_[4드]

- To create Reference data for normal subjects [54]

- To compare gait in patients with fibromyalgia with controls [47]

- To assess gait parameters in children[ $\overline{-}$ - 7 ]

- To discriminate between fallers and non-fallers [48]

- To investigate trunk stability during dual-task walking among older adults $\overline{1} \overline{167]}$

- To investigate the effects of shoe fit on gait in community-dwelling older adults [168]

- To compare the CoM movement within Parkinson's disease (PD) $[\overline{7} 1]$

- To compare gait impairment of patients with neurological condition to healthy subjects [169]

- To compare gait parameters in elderly people with mild cognitive impairment $(\overline{\mathrm{M}} \overline{\mathrm{CI}}) \overline{\text { and }}$ Alzheimer's disease (AD) patients [59]

To investigate the relation of balance to step width variability[58]

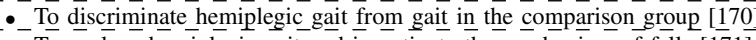

- To analyse hemiplegia gait and investigate the mechanism of falls [171]

To assess spatiotemporal parameters in amputee gait using Dynaport [64

- To investigate the difference in gait patterns for dementia patients_[172]

- To assess the stability and harmony of gait in children with cerebral palsy_[173]

- To investigate effect of dual-task on gait patterns for detection of high risk of falling [17̄ $\overline{7}]$

- To evaluate gait events for_Hemiplegic patients [ $[\overline{6} 6]$

- To distinguish the step events in normal and pathological populations $[1 \overline{17} \overline{5}]$

- To examine the changes in parameters after the drug administration in horses [176]

- To examine the effect of Cueing Strategies on Gait Stability in PD patients [1]7̄]

- To describe the characteristics of stroke patient gait [17 78

- To differentiate between fit and frail older persons [43]

- - To investigate Gait variability_and regularity of people_with transtibial amputations_[1779]

- To investigate the effects of age and gender on gait parameters [3]

To monitor gait of the orthopaedic patients with symptomatic gonarthrosis aimed for total knee arthroplasty [50]

- ${ }^{-}$To investigate the ability to differentiate functional knee $\overline{\text { limitations }} \overline{-} \overline{[180]}$

- To investigate suitability of objective gait measurements related to fall risk based on Tinetti scale [181]

- To assess the effect of prescription footwear on gait quality for gait rehabilitation $\overline{[60]}-$

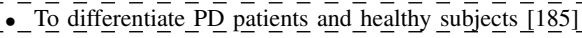

- To differentiate spatio-temporal gait parameters between young and elderly subjects [55]

- To evaluate a mobility assistance system from healthy people and patients[187]

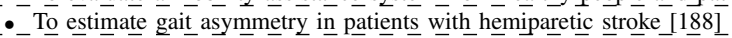

- To quantify movement symmetry in the trotting horse [189]

- To quantitatively investigate whether unexpected gait initiation aggravates $\overline{\text { FoG }} \overline{-}-\overline{-}-\overline{-}-\overline{-}$ with advanced juvenile parkinsonism [190]

To compare gait parameters between two patient groups (Dementia and PD patients) [191]

To investigate relationships between muscle impairments, gait abnormalities and postural instability [192]

- To monitor recovery of gait balance control following concussion [19 $\overline{19} 3]$

- To compare various method for gait parameter estimation using elderly, post stroke, $\overline{\mathrm{PD}} \overline{\text { and }}$ Huntington's disease subjects [194]

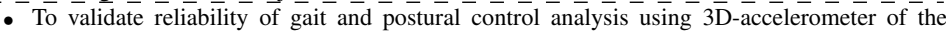
iPod Touch [144]

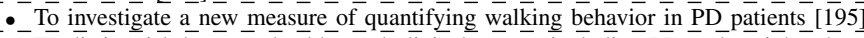

- To distinguish between healthy and clinical groups including $\overline{P D}$ and peripheral neuropathy (PN) patients using time, frequency and time-frequency domains[196]

- To investigate the gait patterns in post-menopausal women [197]

- To evaluate the effect of age, gender and height in gait parameters of healthy subjects $\overline{-} \overline{19} \overline{9} \bar{c}$

of gait parameters across both control subjects and individuals with cerebral palsy.

The gait study has not been limited to humans as there are many applications for analysis of animal gaits. Some examples are quantification of hoof slip distances at training trot [110], detection of unilateral hind limb lameness and foot pathologies in dairy cows [118] and measurement of temporal gait characteristics in dogs [128].

In a sport related application, the relationship between immersion depth and impact loading has been investigated to diminish the injury risk [107] using running trials on the treadmill in three different water depths.

1) Fall and balance analysis: Freezing of Gait (FoG) which is one of the most common causes of fall in PD patients has
- To investigate the relationship of spatiotemporal gait parameters with increasing age $\overline{-1} \overline{1} \overline{6} \overline{6}]$ 
been related to the double support time [69]. Based on this study, gait asymmetries and FoG are evaluated using the gait data from PD patients. It is expected that the detection of subtle changes in FoG session will reduce the risk of falls. Balance and gait deficits in people with multiple sclerosis has also been investigated in [131].

Other papers addressing gait and balance include analysis aimed to discriminate between fallers and non-fallers [48], analyse the gait and balance in patients with Alzheimer's disease [127], evaluate age related gait and balance performance [51], identify gait differences between single-task and dualtask walking in older individuals [109], detect early signs of balance deficits [81], and evaluate the dynamic instability of the dysplastic hip [102]. Hip instability is increased in proportion to the degree of dysplasia. Triaxial accelerometry has shown to be helpful in the evaluation of dynamic instability of the dysplastic hip. The vertical toe clearance during walking has been analyzed in [101]. It has been demonstrated that the variability in minimum toe clearance (MTC), which occurs during the swing phase, is a sensitive fall risk predictor.

\section{Trunk accelerometry based gait analysis}

There has been a great interest in the use of single trunk mounted accelerometer to estimate the spatio-temporal gait parameters and apply it for clinical applications. Table II summarises the gait parameters and the related clinical applications when an IMU has been used as the trunk mounted accelerometer. It should be noted that these studies include the use of accelerometer embedded within smartphones. For a few studies, the gait parameters are estimated using only the gyroscope unit of the IMU and balance analysis are performed using the accelerometer sensor signals. Although it has been noted in Section V-B, the accelerometer can be used to estimate the spatio-temporal gait parameters including step/stride length using the inverted pendulum model.

The trunk mounted accelerometers have been used to investigate the gait patterns of chronic obstructive pulmonary disease (COPD) patients versus healthy subjects [45], create reference data for normal subjects [54], compare gaits in patients with fibromyalgia with those in controls using Locometrix system [47], assess gait parameters in children [57], investigate Gait variability and regularity of people with transtibial amputation [179], compare the CoM movement within PD patients [71], compare gait impairment of patients with neurological condition (including PD and ataxic patients) with those of healthy subjects [169], compare gait parameters in elderly people suffering from mild cognitive impairment (MCI) and Alzheimer's disease (AD) patients using Locometrix system [59], discriminate hemiplegic gait from gait in the comparison group [170], assess spatiotemporal parameters in amputee gait using Dynaport [64], investigate the difference in gait patterns for dementia patients using Dynaport [172], evaluate gait events for Hemiplegic patients [66], distinguish the step events in normal and pathological populations [175], differentiate between fit and frail elderlies [43], describe the characteristics of stroke patient gait [178], differentiate between two groups of young and elderly [184], differentiate spatio-temporal gait parameters between young and old subjects [55], differentiate between PD patients and healthy subjects [185], differentiate $\mathrm{PD}$ and peripheral neuropathy (PN) patients from healthy subjects [196],[199] and validate the estimated stride event versus those by motion capture system [108], investigate the effects of age and gender on gait parameters [3], investigate the effects of age, gender and height on gait parameters [198], estimate gait asymmetry in patients with hemiparetic stroke [188], investigate the relationship between spatio-temporal gait parameters with increasing age [186], monitor gait of the orthopaedic patients with symptomatic gonarthrosis aimed for total knee arthroplasty [50], and investigate the ability to differentiate between functional knee limitations and its suitability for clinical applications [180].

Other applications include assessing the effect of prescription footwear on gait quality for gait rehabilitation [60], evaluating a mobility assistance system from healthy people and patients [187], quantitatively investigating whether an unexpected gait initiation aggravates FoG in patients with advanced juvenile parkinsonism [190], comparison of gait parameters between two patient groups (Dementia and PD patients) [191], comparison of various methods for gait parameter estimation using elderly, post stroke, PD, and Huntington's disease subjects [194], objectively assessing abnormal gait in patients with rheumatoid arthritis using a smartphone [183], investigating the effects of shoe fit on gait in communitydwelling older adults [168], detection of stride event exploiting cross-correlation and anisotropy characteristics of the accelerations using healthy and PD patients [200], a new measure of quantifying walking behavior in PD patients based on gait cycle-acceleration relationship [195] and investigating the gait patterns in post-menopausal women [197]. Animal related studies of trunk accelerometry include quantification of movement symmetry in the trotting horse [189] and examination of the changes in parameters after the drug administration in horses [176].

1) Fall and balance analysis by gait trunk accelerometry: The stability and harmony of gait in children with cerebral palsy has been assessed in [173]. The objectives for fall and balance assessment include analysis of hemiplegia gait and investigate the mechanism of falls [171], investigating the suitability of objective gait measurement related to fall risk assessment based on Tinetti scale [181], discriminating between fallers and non-fallers [48], validating reliability of gait and postural control analysis Using 3D-accelerometer of the iPod Touch [144], investigating the relationships between muscle impairments, gait abnormalities and postural instability [192], monitoring the recovery of gait balance control following concussion [193], examination of the effect of cueing strategies on gait stability in PD patients [177], detection of near fall using Dynaport [182], investigating the relation between balance and step width variability [58], studying the effect of dual-task on gait patterns for detection of high risk of falling using Dynaport [174] and trunk stability during dualtask walking among older adults [167].

\section{Discussion AND CONCLUSION}

In this study a comprehensive review of accelerometry based gait analysis has been performed. Extensive review of single 
and multiple based sensor platforms including accelerometers has been performed. Recent applications are shifting towards the use of smaller number of sensors such as using a single ear-worn sensor, trunk mounted accelerometers and mobile phones. Of these devices, only the ear-worn sensor has shown consistent sensor placement. Developed algorithms based on ear-worn sensor can be further adjusted and improved for application to trunk accelerometer and mobile phones.

Certainly, a thorough study of gait benefits a wide community of researchers, users, and care takers in sport, computer games, physical and mental rehabilitation, clinical assessment, survilence, human recognition, modelling, robotics and many other fields. This study also benefits sensor technology developers and those who enthusiastically work on remote patient monitoring. Different strategies of gait assessment using accelerometry opens a wider arena for research particularly for clinical use and human rehabilitation. One big advantage of using accelerometer for gait analysis is that it can be integrated within smart phones and devices accessible to almost everybody around the globe.

\section{ACKNOWLEDGEMENT}

The authors would like to thank Mr Dinesh Nathwani, consultant Orthopaedic surgeon at Charing Cross Hospital, London, UK, for giving useful comments to improve the quality of the paper.

\section{REFERENCES}

[1] T. K. M. Lee, M. Belkhatir, and S. Sanei, "A comprehensive review of past and present vision-based techniques for gait recognition," Multimedia Tools and Applications, vol. 72, no. 3, pp. 2833-2869, 2014.

[2] T. K. M. Lee, M. Belkhatir, P. A. Lee, and S. Sanei, "Nonlinear characterisation of fronto-normal gait for human recognition," $P C M$ '08 Proceedings of the 9th Pacific Rim Conference on Multimedia: Advances in Multimedia Information Processing, pp. 466-475, 2008.

[3] R. Senden, B. Grimm, I. C. Heyligers, H. Savelberg, and K. Meijer, "Acceleration-based gait test for healthy subjects: reliability and reference data," Gait Posture., vol. 30, no. 2, pp. 192-6, 2009.

[4] K. Kompoliti and L. Verhagen, "Encyclopedia of Movement Disorders," Oxford: Academic Press, 2010.

[5] H. A. Sattar, "Fundamentals of pathology," p. 189, 2011.

[6] P. Dayalu and R. L. Albin, "Huntington disease: pathogenesis and treatment," Neurol Clin., vol. 33, no. 1, pp. 101-114, 2015.

[7] S. C. Warby, R. K. Graham, and M. R. Hayden, "Huntington disease," 2014.

[8] "Ninds multiple sclerosis information page," National Institute of Neurological Disorders and Stroke, 2016.

[9] A. Compston and A. Coles, "Multiple sclerosis," Lancet, vol. 372, no. 9648, pp. 1502-1517, 2008

[10] R. S. Fisher et. al, "Ilae official report: a practical clinical definition of epilepsy," Epilepsia., vol. 55, no. 4, pp. 475-482, 2014.

[11] T. K. M. Lee, S. S. W. Gan, S. Sanei, and S. Kouchaki, "Assessing rehabilitative reach and grasp movements with singular spectrum analysis," 21st European Signal Processing Conference (EUSIPCO 2013), pp. 1-5, 2013.

[12] M. Norris, R. Anderson and I. C. Kenny, "Method analysis of accelerometers and gyroscopes in running gait: a systematic review," Proc. Inst. Mech. Eng. P J. Sports Eng. Technol., vol. 228, no. 1, pp. 3-15, 2014.

[13] R. P. Hubble, G. A. .Naughton , P. A. Silburn, and M. H. Cole, "Wearable sensor use for assessing standing balance and walking stability in people with Parkinson's disease: a systematic review," PLoS One, vol. 10, no. 4, pp. e0123705, 2015.
[14] S. Del Din, A. Hickey, N. Hurwitz, J. C. Mathers, L. Rochester and A. Godfrey, "Measuring gait with an accelerometer-based wearable: influence of device location, testing protocol and age," Physiol Meas., vol. 37 , no. 10 , pp. $1785-1797,2016$.

[15] M. Derawi, "Accelerometer-Based Gait Analysis, A survey," Nor Informasjonssikkerhetskonferanse NISK., 2010.

[16] S. Yang and Q. Li, "Inertial Sensor-Based Methods in Walking Speed Estimation: A Systematic Review," Sensors, vol. 12, no. 5, pp. 61026116, 2012.

[17] S. Chen, J. Lach, B. Lo and G. .Z. Yang, "Toward Pervasive Gait Analysis With Wearable Sensors: A Systematic Review.," IEEE J Biomed Health Inform., vol. 20, no. 6, pp. 1521-1537, 2016.

[18] A. Muro-de-la-Herran, B. Garcia-Zapirain and A. Mendez-Zorrilla, "Gait analysis methods: an overview of wearable and non-wearable systems, highlighting clinical applications.," Sensors, vol. 14, no. 2, pp. 3362-3394, 2014.

[19] S. Sprager and M. B. Juric, "Inertial Sensor-Based Gait Recognition: A Review," Sensors, vol. 15, no. 9, pp. 22089-22127, 2015.

[20] R. J. Elble, "Gravitational artifact in accelerometric measurements of tremor," Clin Neurophysiol., vol. 116, no. 7, pp. 1638-1643, 2005.

[21] B. E. Boser and R. T. Howe, "Surface micromachined accelerometers," IEEE Journal of Solid-State Circuits, vol. 31, no. 3, 1996.

[22] Analog Devices, "ADXL50 datasheet," .

[23] T. L. Grigorie, "The matlab/simulink modeling and numerical simulation of an analogue capacitive micro-accelerometer. part 1: Open loop,' International Conference on Perspective Technologies and Methods in MEMS Design, pp. 105-114, 2008.

[24] I. Skog, P. Hndel, J. O. Nilsson, and J. Rantakokko, "Zero-velocity detection - an algorithm evaluation," IEEE Trans Biomed Eng., vol. 57, no. 11, pp. 2657-2666, 2010.

[25] A. Kos, S. Tomazic and A. Umek, "Suitability of smartphone inertial sensors for real-time biofeedback applications," Sensors, vol. 16, no. 3, pp. 301, 2016.

[26] Q. Mourcou, A. Fleury, C. Franco, F. Klopcic and N. Vuillerme, "Performance evaluation of smartphone inertial sensors measurement for range of motion," Sensors, vol. 15, no. 9, pp. 23168-23187, 2015.

[27] T. Kaiyu and H. G. Malcolm, "A practical gait analysis system using gyroscopes," Med Eng Phys., vol. 21, no. 2, pp. 87-94, 1999.

[28] E. Hestnes, "Performance Evaluation of Smartphone Inertial Sensors Measurement for Range of Motion," NTNTU, Master thesis, 2016.

[29] J. Geen and D. Krakauer, "New iMEMS angular-rate-sensing gyroscope," Analog Dialogue, 2003.

[30] A. Belli, P. Bui, A. Berger, A. Geyssant, and J. R. Lacour, "A treadmill ergometer for three-dimensional ground reaction forces measurement during walking," J Biomech., vol. 34, no. 1, pp. 105-112, 2001.

[31] L. Tesio, M. Monzani, R. Gatti, and F. Franchignoni, "Flexible electrogoniometers: kinesiological advantages with respect to potentiometric goniometers," Clin Biomech., vol. 10, no. 5, pp. 275-277, 1995.

[32] M. Donno, E. Palange, F. Di Nicola, G. Bucci, and F. Ciancetta, "A new flexible optical fiber goniometer for dynamic angular measurements: Application to human joint movement monitoring," IEEE Trans on Instrumentation and Measurement, vol. 57, no. 8, pp. 1614-1620, 2008.

[33] G. S. Murley, H. B. Menz, and K. B. Landorf, "Foot posture influences the electromyographic activity of selected lower limb muscles during gait," J Foot Ankle Res., vol. 2, no. 35, 2009.

[34] A. Hadi, A. Razak, A. Zayegh, R. K. Begg, and Y. Wahab, "Foot plantar pressure measurement system: A review," Sensors, vol. 12, no. 7, pp. 9884-9912, 2012.

[35] S. M. De Rossi, T. Lenzi, N. Vitiello, M. Donati, A. Persichetti, F. Giovacchini, F. Vecchi, and M. C. Carrozza, "Development of an in-shoe pressure-sensitive device for gait analysis.," Conf Proc IEEE Eng Med Biol Soc., pp. 5637-40, 2011.

[36] “MC10," https://www.mc10inc.com/our-products/biostamprc.

[37] "Shimmer Products," http://www.shimmersensing.com/products/.

[38] "Zigbee Health Care," http://www.zigbee.org/zigbee-fordevelopers/applicationstandards/zigbee-health-care/.

[39] J. A. Afonso, A. J. F. Maio, and R. Simoes, "Performance Evaluation of Bluetooth Low Energy for High Data Rate Body Area Networks," Wireless Personal Communications, vol. 90, no. 1, pp. 121-141, 2016.

[40] J. Perry and J. Burnfield, "Gait analysis: Normal and pathological function monitoring," 2nd Ed. SLACK Incorporated, 2010.

[41] H. B. Menz, S. R. Lord, and R. C. Fitzpatrick, "Acceleration patterns of the head and pelvis when walking on level and irregular surfaces," Gait Posture., vol. 18, no. 1, pp. 35-46, 2003.

[42] H. B. Menz, S. R. Lord, and R. C. Fitzpatrick, "Age-related differences in walking stability," Age Ageing., vol. 32, no. 2, pp. 137-142, 2003. 
[43] R. Moe-Nilssen and J. L. Helbostad, "Interstride trunk acceleration variability but not step width variability can differentiate between fit and frail older adults," Gait Posture., vol. 21, no. 2, pp. 164-170, 2005.

[44] A. Hartmann, S. Luzi, K. Murer, R. A. de Bie, and E. D. de Bruin, "Concurrent validity of a trunk tri-axial accelerometer system for gait analysis in older adults," Gait Posture., vol. 29, no. 3, pp. 444-448, 2009.

[45] J. Annegarn, M. A. Spruit, H. H. Savelberg, P. J. Willems, C. van de Bool, A. M. Schols, E. F. Wouters, and K. Meijer, "Differences in walking pattern during 6-min walk test between patients with COPD and healthy subjects," PLoS One., vol. 7, no. 5, 2012.

[46] R. Moe-Nilssen and J. L. Helbostad, "Estimation of gait cycle characteristics by trunk accelerometry," J Biomech., vol. 37, no. 1 , pp. 121-126, 2004.

[47] B. Auvinet, R. Bileckot, A. S. Alix, D. Chaleil, and E. Barrey, "Gait disorders in patients with fibromyalgia," Joint Bone Spine., vol. 73, no. 5, pp. 543-546, 2006.

[48] E. P. Doheny, B. R. Greene, T. Foran, C. Cunningham, C. W. Fan, and R. A. Kenny, "Diurnal variations in the outcomes of instrumented gait and quiet standing balance assessments and their association with falls history," Physiol Meas., vol. 33, no. 3, pp. 361-373, 2012.

[49] F. Bugan, M. G. Benedetti, G. Casadio, S. Attala, F. Biagi, M. Manca, and A. Leardini, "Estimation of spatial-temporal gait parameters in level walking based on a single accelerometer: Validation on normal subjects by standard gait analysis," Comput Methods Programs Biomed, vol. 108, no. 1, pp. 129-137, 2012.

[50] R. Senden, B. Grimm, K. Meijer, H. Savelberg, and I. C. Heyligers, "The importance to including objective functional outcomes in the clinical follow up of total knee arthroplasty patients," Knee., vol. 18, no. 5, pp. 306-11, 2011.

[51] K. Aminian, B. Mariani, A. Paraschiv-Ionescu, C. Hoskovec, C. Bula, J. Penders, C. Tacconi, and F. Marcellini, "Foot worn inertial sensors for gait assessment and rehabilitation based on motorized shoes," Conf Proc IEEE Eng Med Biol Soc., pp. 5820-5823, 2011.

[52] H. Martin Schepers E. H. van Asseldonk C. T. Baten P. H. Veltink, "Ambulatory estimation of foot placement during walking using inertial sensors," J Biomech., vol. 43, no. 16, pp. 3138-3143, 2010.

[53] A. Dalton, H. Khalil, M. Busse, A . Rosser, R. van Deursen, and G. laighin, "Analysis of gait and balance through a single triaxial accelerometer in presymptomatic and symptomatic huntington's disease," Gait Posture., vol. 37, no. 1, pp. 49-54, 2013.

[54] B. Auvinet, G. Berrut, C. Touzard, L. Moutel, N. Collet, D. Chaleil, and E. Barrey, "Reference data for normal subjects obtained with an accelerometric device," Gait Posture, vol. 16, no. 2, pp. 124-134, 2002.

[55] W. Zijlstra, "Assessment of spatio-temporal parameters during unconstrained walking.," Eur J Appl Physiol., vol. 92, no. (1-2), pp. 39-44, 2004

[56] W. Zijlstra and A. L. Hof, "Assessment of spatio-temporal gait parameters from trunk accelerations during human walking," Gait Posture., vol. 18, no. 2, pp. 1-10, 2003.

[57] M. Brandes, W. Zijlstra, S. Heikens, R. van Lummel, and D. Rosenbaum, "Accelerometry based assessment of gait parameters in children," Gait Posture, vol. 24, no. 4, pp. 482-486, 2006.

[58] J. L. Helbostad and R. Moe-Nilssen, "The effect of gait speed on lateral balance control during walking in healthy elderly," Gait Posture., vol. 18 , no. 2 , pp. 27-36, 2003.

[59] S. Gillain, E. Warzee, F. Lekeu, V. Wojtasik, D. Maquet, J. L. Croisier, E. Salmon, and J. Petermans, "The value of instrumental gait analysis in elderly healthy, MCI or alzheimer's disease subjects and a comparison with other clinical tests used in single and dual-task conditions," Ann Phys Rehabil Med., vol. 52, no. 6, pp. 453-474, 2009.

[60] P. Terrier, O. Driaz, A. Meichtry, and F. Luthi, "Prescription footwear for severe injuries of foot and ankle: effect on regularity and symmetry of the gait assessed by trunk accelerometry," Gait Posture., vol. 30, no. 4, pp. 492-496, 2009.

[61] J. B. Bussmann, K. M. Culhane, H. L. Horemans, G. M. Lyons, and H. J. Stam, "Validity of the prosthetic activity monitor to assess the duration and spatio-temporal characteristics of prosthetic walking," IEEE Trans Neural Syst Rehabil Eng., vol. 12, no. 4, pp. 379-386, 2004.

[62] R. C. Gonzalez, D. Alvarez, A. M. Lopez, and J. C. Alvarez, "Ambulatory estimation of mean step length during unconstrained walking by means of COG accelerometry," Comput Methods Biomech Biomed Engin., vol. 12, no. 6, pp. 721-726, 2009.

[63] J. Han, H. S. Jeon, W. J. Yi, B. S. Jeon, and K. S. Park, "Adaptive windowing for gait phase discrimination in parkinsonian gait using 3- axis acceleration signals," Med Biol Eng Comput., vol. 47, no. 11, pp. 1155-1164, 2009.

[64] H. Houdijk, F. M. Appelman, J. M. Van Velzen, L. H. Van der Woude, and C. A. Van Bennekom CA, "Validity of dynaport gaitmonitor for assessment of spatiotemporal parameters in amputee gait," $J$ Rehabil Res Dev., vol. 45, no. 9, pp. 1335-1342, 2008.

[65] M. Boutaayamou, C. Schwartz, J. Stamatakis, V. Denol, D. Maquet, B. Forthomme, J. L. Croisier, B. Macq, J. G. Verly, G. Garraux, and $\mathrm{O}$. Brls, "Development and validation of an accelerometer-based method for quantifying gait events," Med Eng Phys., vol. 37, no. 2, pp. 226-232, 2015.

[66] H. K. Lee, S. J. Hwang, S. P. Cho, D. R. Lee DR, S. H. You, K. J. Lee, Y. H. Kim, and H. S. Choi, "Novel algorithm for the hemiplegic gait evaluation using a single 3-axis accelerometer," Conf Proc IEEE Eng Med Biol Soc., pp. 3964-3966, 2009.

[67] S. W. Lee, K. Mase, and K. Kogure, "Detection of spatio-temporal gait parameters by using wearable motion sensors," Conf Proc IEEE Eng Med Biol Soc., vol. 7, pp. 6836-6839, 2005.

[68] K. J. Donovan, B. R. Greene, D. McGrath, R. O'Neill, A. Burns, and B. Caulfield, "Shimmer: A new tool for temporal gait analysis.," Conf Proc IEEE Eng Med Biol Soc., pp. 3826-3829, 2009.

[69] J. Stamatakis, J. Crmers, D. Maquet, B. Macq, and G. Garraux, "Gait feature extraction in parkinson's disease using low-cost accelerometers," Conf Proc IEEE Eng Med Biol Soc., pp. 7900-7903, 2011.

[70] M. R. Patterson and B. Caulfield, "Using a foot mounted accelerometer to detect changes in gait patterns," Conf Proc IEEE Eng Med Biol Soc., pp. 7471-7475, 2013.

[71] P. Esser, H. Dawes, J. Collett, M. G. Feltham, and K. Howells, "Validity and inter-rater reliability of inertial gait measurements in parkinson's disease: a pilot study," J Neurosci Methods., vol. 205, no. 1, pp. 177 $181,2012$.

[72] E. Sejdic, K. A. Lowry, J. Bellanca, S. Perera, M. S. Redfern, and J. S. Brach, "Extraction of stride events from gait accelerometry during treadmill walking," IEEE J Transl Eng Health Med., vol. 4, 2016.

[73] J. Lasenby and A. Stevenson, "Using geometric algebra for optical motion capture," E. Bayro-Corrochano, G. Sobcyzk (Eds.), Applied Clifford Algebras in Computer Science and Engineering, 2000.

[74] R. Williamson and B. J. Andrews, "Gait event detection for FES using accelerometers and supervised machine learning," IEEE Trans Rehabil Eng., vol. 8, no. 3, pp. 312-319, 2000.

[75] S. Khandelwal and N. Wickstrm, "Evaluation of the performance of accelerometer-based gait event detection algorithms in different realworld scenarios using the MAREA gait database," Gait Posture., vol. 51, pp. 84-90, 2017.

[76] A. Barrett, M. O'Connor, K. Culhane, A. M. Finucane, E. Mulkerrin, D. Lyons, and G. Olaighin, "Accelerometer versus footswitch evaluation of gait unsteadiness and temporal characteristics of gait in two elderly patient groups," Conf Proc IEEE Eng Med Biol Soc, pp. 45274530, 2008.

[77] J. J. Kavanagh, R. S. Barrett, and S. Morrison, "Upper body accelerations during walking in healthy young and elderly men," Gait Posture., vol. 20, no. 3, pp. 291-298, 2004.

[78] A. Kose, A. Cereatti, and U. Della Croce, "Estimation of traversed distance in level walking using a single inertial measurement unit attached to the waist," Conf Proc IEEE Eng Med Biol Soc., pp. 11251128, 2011.

[79] J. A. Lee, S. H. Cho, Y. J. Lee, H. K. Yang, and J. W. Lee, "Portable activity monitoring system for temporal parameters of gait cycles," $J$ Med Syst., vol. 34, no. 5, pp. 959-966, 2010.

[80] D. Jarchi, B. Lo, E. Ieong, D. Nathwani, and G.-Z. Yang, "Validation of the e-AR sensor for gait event detection using the Parotec foot insole with application to post-operative recovery monitoring," 11th International Conference on Wearable and Implantable Body Sensor Networks (BSN), pp. 127-131, 2014.

[81] A. Hartmann, K. Murer, R. A. de Bie, and E. D. de Bruin ED., "Reproducibility of spatio-temporal gait parameters under different conditions in older adults using a trunk tri-axial accelerometer system," Gait Posture., vol. 30, no. 3, pp. 351-355, 2009.

[82] I. H. Lpez-Nava, A. Muoz-Melndez, A. I. Prez Sanpablo, A. Alessi Montero, I. Quiones Uristegui, and L. Nez Carrera, "Estimation of temporal gait parameters using bayesian models on acceleration signals," Comput Methods Biomech Biomed Engin., vol. 19, no. 4, pp. 396-403, 2016.

[83] S. Y. Lim and W. H. Lee, "Comparison of accelerometer-based and treadmill-based analysis systems for measuring gait parameters in healthy adults," J Phys Ther Sci., vol. 29, no. 4, pp. 651-653, 2017. 
[84] E. Bergamini, P. Picerno, H. Pillet, F. Natta, P. Thoreux, and V. Camomilla, "Estimation of temporal parameters during sprint running using a trunk-mounted inertial measurement unit," J Biomech., vol. 45, no. 6, pp. 1123-1126, 2012.

[85] M. Hanlon and R. Anderson, "Real-time gait event detection using wearable sensors," Gait Posture., vol. 30, no. 4, pp. 523-527, 2009.

[86] N. A. Maffiuletti, M. Gorelick, I. Kramers de Quervain, M. Bizzini, S. Tomasetti J. P. Munzinger JP, and A. Stacoff, "Concurrent validity and intrasession reliability of the IDEEA accelerometry system for the quantification of spatiotemporal gait parameters," Gait Posture., vol. 27, no. 1, pp. 160-163, 2008.

[87] Y. Sakurai, Y. Ohgi, and T. Maruyama, "Development and validation of an automated method to detect gait events using acceleration and angular rate (p216)," The Engineering of Sport 7, pp. 359-366, 2009.

[88] R. W. Selles, M. A. Formanoy, J. B. Bussmann, P. J. Janssens, and H. J. Stam HJ., "Automated estimation of initial and terminal contact timing using accelerometers; development and validation in transtibial amputees and controls," IEEE Trans Neural Syst Rehabil Eng., vol. 13 , no. 1 , pp. 81-88, 2005.

[89] J. Sinclair, S. J. Hobbs, L. Protheroe, C. J. Edmundson, and A. Greenhalgh, "Determination of gait events using an externally mounted shank accelerometer.," J Appl Biomech., vol. 29, no. 1, pp. 118-122, 2013.

[90] D. Jarchi, C. Wong, R. Kwasnicki, B. Heller, G. Tew, and G.-.Z. Yang, "Gait parameter estimation from a miniaturized ear-worn sensor using singular spectrum analysis and longest common subsequence," IEEE Trans Biomed Eng, vol. 61, no. 4, pp. 1261-1273, 2014.

[91] L. Atallah, A. Wiik, G. G. Jones, B. Lo, J. P. Cobb, A. Amis, and G. Z. Yang, "Validation of an ear-worn sensor for gait monitoring using a force-plate instrumented treadmill," Gait Posture., vol. 35, no. 4, pp. 674-676, 2012.

[92] K. Ben Mansour, N. Rezzougand, and P. Gorce, "Analysis of several methods and inertial sensors locations to assess gait parameters in ablebodied subjects," Gait Posture., vol. 42, no. 4, pp. 409-414, 2015.

[93] O. Tupa, A. Prochazka, O. Vysata, M. Schatz, J. Mares, M. Valis and V. Marik, "Motion tracking and gait feature estimation for recognising Parkinson's disease using MS Kinect," Biomed Eng Online., vol. 14, no. 97,2015

[94] A. Prochazka, O. Vysata, M. Valis, O. Tupa, M. Schatz and V. Marik, "Bayesian classification and analysis of gait disorders using image and depth sensors of Microsoft Kinect," Digital Signal Processing, vol. 47, pp. 169-177, 2015.

[95] M. .L. Gorelick, M. Bizzini, N. A. Maffiuletti, J. .P. Munzinger, and U. Munzinger, "Test-retest reliability of the IDEEA system in the quantification of step parameters during walking and stair climbing," Clin Physiol Funct Imaging., vol. 29, no. 4, pp. 271-276, 2009.

[96] P. E. Shrout and J. L. Fleiss, "Intraclass correlations: uses in assessing rater reliability," Psychol Bull., vol. 86, no. 2, pp. 420-428, 1979.

[97] D. Jarchi, A. Peters, B. Lo, E. Kalliolia, I. Di. Giulio, P. Limousin, B. L. Day, and G.-Z. Yang, "Assessment of the e-ar sensor for gait analysis of parkinson's disease patients," IEEE 12th International Conference on Wearable and Implantable Body Sensor Networks (BSN),, pp. 1-6, 2015.

[98] D. Jarchi, B. Lo, C. Wong, E. Ieong, D. Nathwani, and G.-Z. Yang, "Gait analysis from a single ear-worn sensor: Reliability and clinical evaluation for orthopaedic patients," IEEE Trans Neural Syst Rehabil Eng., vol. 24, no. 8, pp. 882-892, 2016.

[99] R. M. Kwasnicki, S. Hettiaratchy, D. Jarchi, C. Nightingale, M. Wordsworth, J. Simmons, G.-Z. Yang, and A. Darzi, "Assessing functional mobility after lower limb reconstruction: A psychometric evaluation of a sensor-based mobility score," Annals of Surgery, vol. 261, no. 4, pp. 800-806, 2015.

[100] L. Atallah, A. Wiik, B. Lo, J. P. Cobb, A. A. Amis, and G. Z. Yang, "Gait asymmetry detection in older adults using a light earworn sensor," Physiol Meas., vol. 35, no. 5, pp. N29-40, 2014.

[101] D. T. H. Lai, R. Begg, E. Charry M. Palaniswami, and K. Hill, "Measuring toe clearance using a wireless inertial sensing device," International Conference on Intelligent Sensors, Sensor Networks and Information Processing, Sydney, NSW, pp. 375-380, 2008.

[102] A. Maeyama, M. Naito, S. Moriyama, and I. Yoshimura, "Evaluation of dynamic instability of the dysplastic hip with use of triaxial accelerometry," J Bone Joint Surg Am., vol. 90, no. 1, pp. 85-92, 2008.

[103] S. Park, K. Ryu, J. Kim, J. Son, and Y. Kim, "Verification of accuracy and validity of gait phase detection system using motion sensors for applying walking assistive FES," Comput Methods Biomech Biomed Engin., vol. 15, no. 11, pp. 1129-1135, 2012.
[104] G. Pendharkar, P. Percival, D. Morgan, and D. Lai, "Automated method to distinguish toe walking strides from normal strides in the gait of idiopathic toe walking children from heel accelerometry data," Gait Posture., vol. 35, no. 3, pp. 478-482, 2012.

[105] Y. Shimada, S. Ando, T. Matsunaga, A. Misawa, T. Aizawa, T. Shirahata, and E. Itoi, "Clinical application of acceleration sensor to detect the swing phase of stroke gait in functional electrical stimulation," Tohoku J Exp Med., vol. 207, no. 3, pp. 197-202, 2005.

[106] A. Tura, L. Rocchi, M. Raggi, A. G. Cutti, and L. Chiari, "Toward a virtual gait trainer: gait regularity assessment in transfemoral amputees by on-body accelerometers and gyroscopes," Virtual Rehabilitation, pp. 78-78, 2007

[107] P. W. Macdermid, J. Wharton, C. Schill, and P. W. Fink, "Water depth effects on impact loading, kinematic and physiological variables during water treadmill running," Gait Posture., vol. 56, pp. 108-111, 2017.

[108] H. F. Maqbool, M. A. B. Husman, M. I. Awad, A. Abouhossein, P. Mehryar, N. Iqbal, and A. A. Dehghani-Sanij, "Real-time gait event detection for lower limb amputees using a single wearable sensor," 38th Annual International Conference of the IEEE Engineering in Medicine and Biology Society (EMBC), pp. 5067-5070., 2016.

[109] J. D. Howcroft, E. D. Lemaire, J. Kofman, and W. E. McIlroy, "Analysis of dual-task elderly gait using wearable plantar-pressure insoles and accelerometer," Conf Proc IEEE Eng Med Biol Soc., pp. 5003-5006, 2014.

[110] L. Holden-Douilly, P. Pourcelot, L. Desquilbet, S. Falala, N. CrevierDenoix, and $\mathrm{H}$. Chateau, "Equine hoof slip distance during trot at training speed: comparison between kinematic and accelerometric measurement techniques," Vet J., vol. 197, no. 2, pp. 198-204, 2013.

[111] R. Ganea, P. V. Jeannet, A. Paraschiv-Ionescu, N. M. Goemans, C. Piot, M. Van den Hauwe, and K. Aminian, "Gait assessment in children with duchenne muscular dystrophy during long-distance walking," J Child Neurol., vol. 27, no. 1, pp. 30-8, 2012.

[112] J. M. Jasiewicz, J. H. Allum, J. W. Middleton, A. Barriskill, P. Condie, B. Purcell, and R. C. Li, "Gait event detection using linear accelerometers or angular velocity transducers in able-bodied and spinal-cord injured individuals," Gait Posture., vol. 24, no. 4, pp. 502-509, 2006.

[113] M. D. Latt, H. B. Menz, V. S. Fung, and S. R. Lord, "Acceleration patterns of the head and pelvis during gait in older people with parkinson's disease: a comparison of fallers and nonfallers," J Gerontol A Biol Sci Med Sci., vol. 64, no. 6, pp. 700-706, 2009.

[114] H. B. Menz, S. R. Lord, S. T. George. R, and R. C. Fitzpatrick, "Walking stability and sensorimotor function in older people with diabetic peripheral neuropathy," Arch Phys Med Rehabil., vol. 85, no. 2, pp. 245-252, 2004.

[115] M. Sekine, Y. Abe, M. Sekimoto, Y. Higashi, T. Fujimoto, T. Tamura, and Y. Fukui, "Assessment of gait parameter in hemiplegic patients by accelerometry," Proceedings of the 22nd Annual International Conference of the IEEE Engineering in Medicine and Biology Society (Cat. No.00CH37143), vol. 3, pp. 1879-1882, 2000.

[116] R. R. Torrealba, J. Cappelletto, L. Fermin-Leon, J. C. Grieco, and G. Fernandez-Lopez, "Statistics-based technique for automated detection of gait events from accelerometer signals," Electronics Letters, vol. 46, no. 22, pp. 1483-1485, 2010.

[117] H. Simil, M. Immonen, and M. Ermes, "Accelerometry-based assessment and detection of early signs of balance deficits," Comput Biol Med., vol. 85, pp. 25-32, 2017.

[118] M. Alsaaod, M. Luternauer, T. Hausegger, R. Kredel, and A. Steiner, "The cow pedogram-analysis of gait cycle variables allows the detection of lameness and foot pathologies," J Dairy Sci., vol. 100, no. 2, pp. 1417-1426, 2017.

[119] A. Khan and E. Biddiss, "Musical stairs: A motivational therapy tool for children with disabilities featuring automated detection of stairclimbing gait events via inertial sensors," Med Eng Phys., vol. 40, pp. 95-102, 2017.

[120] A. Rampp, J. Barth, S. Schlein, K. G. Gamann, J. Klucken, and B. M Eskofier, "Inertial sensor-based stride parameter calculation from gait sequences in geriatric patients," IEEE Trans Biomed Eng., vol. 62, no. 4, pp. 1089-1097, 2015.

[121] S. Reinfelder, R. Hauer, J. Barth, J. Klucken, and B. M. Eskofier, "Timed Up-and-Go phase segmentation in parkinson's disease patients using unobtrusive inertial sensors," Conf Proc IEEE Eng Med Biol Soc., pp. 5171-5174, 2015.

[122] A. Brgou Bourgeois, B. Mariani, K. Aminian, P. Y. Zambelli, and C. J. Newman, "Spatio-temporal gait analysis in children with cerebral palsy using, foot-worn inertial sensors," Gait Posture., vol. 39, no. 1, pp. 436-442, 2014. 
[123] U. R. Acharya, S. V. Sree, C. M. Lim, P. C. Ang, M. Sekine, and T. Tamura, "Comparison of walking parameters obtained from the young, elderly and adults with support," Comput Methods Biomech Biomed Engin., vol. 16, no. 11, pp. 1202-1212, 2013.

[124] Y. Guo, D. Wu, G. Liu, G. Zhao, B. Huang, and L. Wang, "A lowcost body inertial-sensing network for practical gait discrimination of hemiplegia patients," Telemed J E Health., vol. 18, no. 10, pp. 748-754, 2012.

[125] H. Lau and K. Tong, "The reliability of using accelerometer and gyroscope for gait event identification on persons with dropped foot," Gait Posture., vol. 27, no. 2, pp. 248-257, 2008.

[126] J. Rueterbories, E. G. Spaich, and O. K. Andersen, "Characterization of gait pattern by 3D angular accelerations in hemiparetic and healthy gait," Gait Posture., vol. 37, no. 2, pp. 183-189, 2013.

[127] Y. L. Hsu, P. C. Chung, W. H. Wang, M. C. Pai, C. Y. Wang, C. W. Lin, H. L. Wu, and J. S. Wang, "Gait and balance analysis for patients with alzheimer's disease using an inertial-sensor-based wearable instrument," IEEE J Biomed Health Inform., vol. 18, no. 6, pp. 1822-1830, 2014.

[128] C. Ladha, J. O'Sullivan, Z. Belshaw, and L. Asher, "Gaitkeeper: A system for measuring canine gait," Sensors (Basel)., vol. 17, no. 2, 2017.

[129] R. Barrois, T. Gregory, L. Oudre, T. Moreau, C. Truong, A. AramPulini, A. Vienne, C. Labourdette, N. Vayatis, S. Buffat, A. Yelnik, C. Waele, S. Laporte, P. P. Vidal, and D. Ricard, "An automated recording method in clinical consultation to rate the limp in lower limb osteoarthritis," PLoS One., vol. 11, no. 10, 2016.

[130] A. H. Mackey, N. S. Stott, and S. E. Walt, "Reliability and validity of an activity monitor (IDEEA) in the determination of temporal-spatial gait parameters in individuals with cerebral palsy," Gait Posture., vol. 28, no. 4, pp. 634-639, 2008.

[131] R. I. Spain, R. J. St. George, A. Salarian, M. Mancini, J. M. Wagner F. B. Horak, and D. Bourdette, "Body-worn motion sensors detect balance and gait deficits in people with multiple sclerosis who have normal walking speed," Gait Posture., vol. 35, no. 4, pp. 573-578, 2012.

[132] S. Tadano, R. Takeda, K. Sasaki, T. Fujisawa, and H. Tohyama, "Gait characterization for osteoarthritis patients using wearable gait sensors (h-gait systems).," J Biomech., vol. 49, no. 5, pp. 684-690, 2016.

[133] V. Maynard, A. M. Bakheit, J. Oldham, and J. Freeman, "Intra-rater and inter-rater reliability of gait measurements with CODA mpx30 motion analysis system," Gait Posture., vol. 17, no. 1, pp. 59-67, 2003.

[134] S. E. Lord, M. Weatherall, and L. Rochester, "Community ambulation in older adults: which internal characteristics are important?," Arch Phys Med Rehabil., vol. 91, no. 3, pp. 378-383, 2010.

[135] S. Hwang G. Moon and Y. Kim, "Is tow-accelerometer set-up better for the determination of gait patterns?," World Congress on Medical Physics and Biomedical Engineering, pp. 2999-3002, 2006.

[136] R. Schwesig, R. Kauert, S. Wust, S. Becker, and S. Leuchte, "Reliability of the novel gait analysis system Rehawatch," Biomed Tech (Berl)., vol. 55 , no. 2 , pp. 109-115, 2010.

[137] R. Lemoyne T. Mastroianni M. Cozza C. Coroian W. Grundfest, "Implementation of an iphone as a wireless accelerometer for quantifying gait characteristics," Conf Proc IEEE Eng Med Biol Soc., pp. 38473851, 2010.

[138] F. Kluge, H. Ganer, J. Hannink, C. Pasluosta, J. Klucken and B. M. Eskofier, "Towards mobile gait analysis: concurrent validity and testretest reliability of an inertial measurement system for the assessment of spatio-temporal gait parameters," Sensors, vol. 17, no. 7, 2017.

[139] L. Donath, O. Faude, E. Lichtenstein, G. Pagenstert, C. Nesch and A. Mndermann, "Mobile inertial sensor based gait analysis: Validity and reliability of spatiotemporal gait characteristics in healthy seniors," Gait Posture, vol. 49, pp. 371-374, 2016.

[140] L. Donath, O. Faude, E. Lichtenstein, C. Nuesch and A. Mundermann, "Validity and reliability of a portable gait analysis system for measuring spatiotemporal gait characteristics: comparison to an instrumented treadmill," Journal of NeuroEngineering and Rehabilitation, vol. 13, no. 6, 2016.

[141] J. S. Brach, S. Perera, S. Studenski and A. B. Newman, "The reliability and validity of measures of gait variability in community-dwelling older adults," Arch Phys Med Rehabil., vol. 89, no. 12, pp. 22932296, 2008.

[142] C. J. van Uden and M. P. Besser, "Test-retest reliability of temporal and spatial gait characteristics measured with an instrumented walkway system (GAITRite)," BMC Musculoskelet Disord., vol. 5, no. 13, 2004.
[143] M. Montero-Odasso, A. Casas, K. T. Hansen, P. Bilski, I. Gutmanis, J. L. Wells and M. J. Borrie, "Quantitative gait analysis under dual-task in older people with mild cognitive impairment: a reliability study," $J$ Neuroeng Rehabil., vol. 6, no. 35, 2009.

[144] N. M. Kosse, S. Caljouw, D. Vervoort, N. Vuillerme, and C. J. Lamoth, "Validity and reliability of gait and postural control analysis using the tri-axial accelerometer of the iPod touch," Ann Biomed Eng., vol. 43, no. 8, pp. 1935-1946, 2015.

[145] T. T. Pham, S. T. Moore, S. J. G. Lewis, D. N. Nguyen, E. Dutkiewicz, A. J. Fuglevand, A. L. McEwan and P. H. W. Leong, "Freezing of gait detection in parkinson's disease: A subject-independent detector using anomaly scores," IEEE Trans Biomed Eng, vol. 64, no. 11, pp. 27192728, 2017.

[146] Z. He and W. Zhang, "Estimation of walking speed using accelerometer and artificial neural networks," Part of the Communications in Computer and Information Science book series (CCIS, volume 159), vol. 159 , pp. 42-47, 2011.

[147] Q. Li, M. Young, V. Naing, and J. M. Donelan, "Walking speed and slope estimation using shank-mounted inertial measurement units," IEEE International Conference on Rehabilitation Robotics, Kyoto International Conference Center, pp. 839-844, 2009.

[148] Y. Song, S. Shin, S. Kim, D. Lee, and K. H. Lee, "Speed estimation from a tri-axial accelerometer using neural networks.," Conf Proc IEEE Eng Med Biol Soc., pp. 3224-3227, 2007.

[149] E. Bishop and Q. Li, "Walking speed estimation using shank-mounted accelerometers," IEEE International Conference on Robotics and Automation, pp. 5096-5101, 2010.

[150] A. Zijlstra and W. Zijlstra, "Trunk-acceleration based assessment of gait parameters in older persons: a comparison of reliability and validity of four inverted pendulum based estimations," Gait Posture., vol. 38, no. 4, pp. 940-944, 2013.

[151] M. Kawabata, K. Goto, C. Fukusaki, K. Sasaki, E. Hihara, T. Mizushina, and N. Ishii, "Acceleration patterns in the lower and upper trunk during running," J Sports Sci., vol. 31, no. 16, pp. 1841$1853,2013$.

[152] C. Little, J. Lee, D. A. James, and K. Davison, "An evaluation of inertial sensor technology in the discrimination of human gait," $J$ Sports Sci., vol. 31, no. 12, pp. 1312-1318, 2013.

[153] J. C. Alvarez, R. C. Gonzlez, D. Alvarez, A. M. Lpez, and J. RodrguezUra, "Multisensor approach to walking distance estimation with foot inertial sensing," Conf Proc IEEE Eng Med Biol Soc., pp. 5719-5722, 2007.

[154] A. Kose, A. Cereatti, and U. Della Croce, "Bilateral step length estimation using a single inertial measurement unit attached to the pelvis," J Neuroeng Rehabil., vol. 9, no. 9, 2012.

[155] Q. Li, M. Young, V. Naing, and J. M. Donelan, "Walking speed estimation using a shank-mounted inertial measurement unit," $J$ Biomech., vol. 43, no. 8, pp. 1640-1643, 2010.

[156] S. Yang and Q. Li, "IMU-based ambulatory walking speed estimation in constrained treadmill and overground walking," Comput Methods Biomech Biomed Engin., vol. 15, no. 3, pp. 313-322, 2012.

[157] S. Yang, A. Laudanski, and Q. Li, "Inertial sensors in estimating walking speed and inclination: an evaluation of sensor error models," Med Biol Eng Comput., vol. 50, no. 4, pp. 383-93, 2012.

[158] S. Gillain, M. Boutaayamou, N. Dardenne, C. Schwartz, M. Demonceau, C. Gerontitis, F. Depierreux, E. Salmon, G. Garraux, O. Bruyre, O. Brls, J. L. Croisier, and J. Petermans, "Data set of healthy old people assessed for three walking conditions using accelerometric and opto-electronic methods," Aging Clin Exp Res., 2017.

[159] C. M. Barnes, C. C. Clark, M. D. Holton, G. Stratton, and H. D. Summers, "Quantitative time profiling of children's activity and motion," Med Sci Sports Exerc., vol. 49, no. 1, pp. 183-190, 2017.

[160] I. Gonzlez, J. Fontecha, R. Hervs, and J. Bravo, "Estimation of temporal gait events from a single accelerometer through the scalespace filtering idea," J Med Syst., vol. 40, no. 12, pp. 251, 2016.

[161] H. Zhou, N. Ji, O. W. Samuel, Y. Cao, Z. Zhao, S. Chen, and G. Li, "Towards real-time detection of gait events on different terrains using time-frequency analysis and peak heuristics algorithm," Sensors (Basel)., vol. 16, no. 10, 2016.

[162] K. H. Schtte, J. Aeles, T. O. De-Beck, B. C. van der Zwaard, R. Venter, and B. Vanwanseele, "Surface effects on dynamic stability and loading during outdoor running using wireless trunk accelerometry," Gait Posture., vol. 48, pp. 220-205, 2016.

[163] N. Kitagawa and N. Ogihara, "Estimation of foot trajectory during human walking by a wearable inertial measurement unit mounted to the foot," Gait Posture., vol. 45, pp. 110-104, 2016. 
[164] K. Btzel, F. M. Marti, M. A. Rodrguez, A. Plate, and A. O. Vicente, "Gait recording with inertial sensors-how to determine initial and terminal contact," J Biomech., vol. 49, no. 3, pp. 332-337, 2016.

[165] L. Pepa, F. Verdini, and L. Spalazzi, "Experimental evaluation of a smartphone based step length estimation," Conf Proc IEEE Eng Med Biol Soc., pp. 7647-7650, 2015.

[166] D. Trojaniello, A. Cereatti, and U. Della Croce, "Accuracy, sensitivity and robustness of five different methods for the estimation of gait temporal parameters using a single inertial sensor mounted on the lower trunk," Gait Posture., vol. 40, no. 4, pp. 487-492, 2014.

[167] T. Doi, H. Makizako, H. Shimada, D. Yoshida, K. Ito, T. Kato, H. Ando, and T. Suzuki, "Brain atrophy and trunk stability during dual-task walking among older adults," J Gerontol A Biol Sci Med Sci., vol. 67, no. 7, pp. 790-795, 2012.

[168] T. Doi, R. Yamaguchi, T. Asai, M. Komatsu, D. Makiura, M. Shimamura, S. Hirata, H. Ando, and M. Kurosaka, "The effects of shoe fit on gait in community-dwelling older adults.," Gait Posture., vol. 32, no. 2, pp. 274-278, 2010.

[169] P. Fazio, G. Granieri, I. Casetta, E. Cesnik, S. Mazzacane, P. Caliandro, F. Pedrielli, and E. Granieri E., "Gait measures with a triaxial accelerometer among patients with neurological impairment," Neurol Sci., vol. 34, no. 4, pp. 435-440, 2013.

[170] C. Hodt-Billington, J. L. Helbostad, and R. Moe-Nilssen, "Should trunk movement or footfall parameters quantify gait asymmetry in chronic stroke patients?," Gait Posture, vol. 27, no. 4, pp. 552-558, 2008

[171] F. Horiuchi, R. Kadoya, Y. Higasi, T. Fujimoto, M. Sekine, and T. Tamura, "Evaluation by accelerometry of walking pattern before falls in hemiplegic patients," 2001 Conference Proceedings of the 23rd Annual International Conference of the IEEE Engineering in Medicine and Biology Society, vol. 2, pp. 1153-1154, 2001.

[172] T. Ijmker and C. J. Lamoth, "Gait and cognition: the relationship between gait stability and variability with executive function in persons with and without dementia," Gait Posture., vol. 35, no. 1, pp. 126-130, 2012.

[173] M. Iosa, T. Marro, S. Paolucci, and D. Morelli, "Stability and harmony of gait in children with cerebral palsy," Res Dev Disabil., vol. 33, no. 1, pp. 129-135, 2012.

[174] C. J. Lamoth, F. J. van Deudekom, J. P. van Campen, B. A. Appels, O. J. de Vries, and M. Pijnappels, "Gait stability and variability measures show effects of impaired cognition and dual tasking in frail people," $J$ Neuroeng Rehabil., vol. 8, no. 2, 2011.

[175] H. K. Lee, J. You, S. P. Cho, S. J. Hwang, D. R. Lee, Y. H. Kim, and K. J. Lee, "Computational methods to detect step events for normal and pathological gait evaluation using accelerometer," Electronics Letters, vol. 46, no. 17 , pp. $1185-1187,2010$.

[176] F. J. Lpez-Sanromn, R. Holmbak-Petersen, I. Santiago, I. A. Gmez de Segura, and E. Barrey, "Gait analysis using $3 \mathrm{~d}$ accelerometry in horses sedated with xylazine," Vet J., vol. 193, no. 1, pp. 212-216, 2012

[177] K. L. Lowry, A. J. Carrel, J. M. McIlrath, and A. L. Smiley-Oyen, "Use of harmonic ratios to examine the effect of cueing strategies on gait stability in persons with parkinson's disease," Arch Phys Med Rehabil., vol. 91, no. 4, pp. 632-638, 2010.

[178] C. Mizuike, S. Ohgi, and S. Morita, "Analysis of stroke patient walking dynamics using a tri-axial accelerometer," Gait Posture., vol. 30, no. 1, pp. 60-64, 2009.

[179] K. Parker, E. Hanada, and J. Adderson, "Gait variability and regularity of people with transtibial amputations," Gait Posture., vol. 37, no. 2, pp. 269-273, 2013.

[180] R. Senden, I. C. Heyligers, K. Meijer, H. Savelberg, and B. Grimm, "Acceleration-based motion analysis as a tool for rehabilitation: exploration in simulated functional knee limited walking conditions," Am J Phys Med Rehabil., vol. 90, no. 3, pp. 226-232, 2011.

[181] R. Senden, H. H. Savelberg, B. Grimm, I. C. Heyligers, and K. Meijer, "Accelerometry-based gait analysis, an additional objective approach to screen subjects at risk for falling," Gait Posture., vol. 36, no. 2, pp. 296-300, 2012.

[182] A. Weiss, I. Shimkin, N. Giladi, and J. M. Hausdorff, "Automated detection of near falls: algorithm development and preliminary results," BMC Res Notes., vol. 3, no. 62, 2010.

[183] M. Yamada, T. Aoyama, S. Mori, S. Nishiguchi, K. Okamoto, T. Ito, S. Muto, T. Ishihara, H. Yoshitomi, and H. Ito, "Objective assessment of abnormal gait in patients with rheumatoid arthritis using a smartphone," Rheumatol Int., vol. 32, no. 12, pp. 3869-3874, 2012.

[184] R. Yamaguchi, S. Hirata, T. Doi, T. Asai, J. Inoue2, D. Makiura, H. Ando, M. Kurosaka, and Y. Miura, "The usefulness of a new gait symmetry parameter derived from lissajous figures of tri-axial acceleration signals of the trunk," Journal of Physical Therapy Science, vol. 24, no. 5, pp. 405-408, 2012.

[185] C. C. Yang, Y. L. Hsu, K. S. Shih, and J. M. Lu, "Real-time gait cycle parameter recognition using a wearable accelerometry system," Sensors (Basel)., vol. 11, no. 8, pp. 7314-7326, 2011.

[186] A. Zijlstra, E. D. de Bruin, N. Bruins, and W. Zijlstra, "The step length-frequency relationship in physically active community-dwelling older women," Eur J Appl Physiol., vol. 104, no. 3, pp. 427-34, 2008.

[187] C. Zong, M. Chetouani, and A. Tapus, "Automatic gait characterization for a mobility assistance system," 11th International Conference on Control Automation Robotics and Vision, Singapore, pp. 473-478, 2010.

[188] K. Oyake, T. Yamaguchi, M. Sugasawa, C. Oda, S. Tanabe, K. Kondo, Y. Otaka, and K. Momose, "Validity of gait asymmetry estimation by using an accelerometer in individuals with hemiparetic stroke," $J$ Phys Ther Sci., vol. 29, no. 2, pp. 307-311, 2017.

[189] T. Pfau and R. Weller, "Comparison of a standalone consumer grade smartphone with a specialist inertial measurement unit for quantification of movement symmetry in the trotting horse," Equine Vet J., vol. 49, no. 1, pp. 124-129, 2017.

[190] M. Ishii and H. Mashimo, "Accelerometer based analysis of gait initiation failure in advanced juvenile parkinsonism: A single subject study," J Phys Ther Sci, vol. 28, no. 11, pp. 3252-3256, 2016.

[191] "Ambulatory gait behavior in patients with dementia: A comparison with parkinson's disease," IEEE Trans Neural Syst Rehabil Eng., vol. 24, no. 8, pp. 817-826, 2016.

[192] D. Bachasson, A. Moraux, G. Ollivier, V. Decostre, I. Ledoux, T. Gidaro, L. Servais, A. Behin, T. Stojkovic, L. J. Hbert, J. Puymirat, B. Eymard, G. Bassez, and J. Y. Hogrel, "Relationship between muscle impairments, postural stability, and gait parameters assessed with lower-trunk accelerometry in myotonic dystrophy type 1," Neuromuscul Disord., vol. 26, no. 7, pp. 428-435, 2016.

[193] D. Howell, L. Osternig, and L. S. Chou, "Monitoring recovery of gait balance control following concussion using an accelerometer," $J$ Biomech., vol. 48, no. 12, pp. 3364-3368, 2015.

[194] D. Trojaniello, A. Ravaschio, J. M. Hausdorff, and A. Cereatti, "Comparative assessment of different methods for the estimation of gait temporal parameters using a single inertial sensor: application to elderly, post-stroke, parkinson's disease and huntington's disease subjects," Gait Posture., vol. 42, no. 3, pp. 310-306, 2015.

[195] M. Yoneyama, Y. Kurihara, K. Watanabe, and H. Mitoma, "Accelerometry-based gait analysis and its application to parkinson's disease assessment- part 2: a new measure for quantifying walking behavior," IEEE Trans Neural Syst Rehabil Eng., vol. 21, no. 6, pp. 999-1005, 2013.

[196] E. Sejdi, K. A. Lowry, J. Bellanca, M. S .Redfern and J. S. Brach, "A comprehensive assessment of gait accelerometry signals in time, frequency and time-frequency domains," IEEE Trans Neural Syst Rehabil Eng., vol. 22, no. 3, 2014.

[197] F. Manikowska, K. Hojan, P. J. Chen, M. Jwiak, and A. Jwiak, "The gait pattern in post-menopausal women. pilot study," Ortop Traumatol Rehabil., vol. 15, no. 6, pp. 575-583, 2013.

[198] R. Senden, K. Meijer, I. C. Heyligers, H. H. Savelberg and B. Grimm, "Importance of correcting for individual differences in the clinical diagnosis of gait disorders," Physiotherapy., vol. 98, no. 4, pp. 320324, 2012.

[199] A. Millecamps, K. A. Lowry, J. S. Brach, S. Perera, M. S. Redfern and E. Sejdi, "Understanding the effects of pre-processing on extracted signal features from gait accelerometry signals.," Comput Biol Med., vol. 62 , pp. 164-174, 2015.

[200] M. Yoneyama, Y. Kurihara, K. Watanabe and H. Mitoma, "Accelerometry-based gait analysis and its application to Parkinson's disease assessment-part 1: detection of stride event," IEEE Trans Neural Syst Rehabil Eng., vol. 22, no. 3, pp. 613-622, 2014. 